\title{
Convection-Reaction Equation Based Magnetic Resonance Electrical Properties Tomography (cr-MREPT)
}

\author{
Fatih S. Hafalir, Omer F. Oran, Necip Gurler, and Yusuf Z. Ider*, Member, IEEE
}

\begin{abstract}
Images of electrical conductivity and permittivity of tissues may be used for diagnostic purposes as well as for estimating local specific absorption rate distributions. Magnetic resonance electrical properties tomography (MREPT) aims at noninvasively obtaining conductivity and permittivity images at radio-frequency frequencies of magnetic resonance imaging systems. MREPT algorithms are based on measuring the B1 field which is perturbed by the electrical properties of the imaged object. In this study, the relation between the electrical properties and the measured $\mathrm{B} 1$ field is formulated for the first time as a well-known convection-reaction equation. The suggested novel algorithm, called "cr-MREPT," is based on the solution of this equation on a triangular mesh, and in contrast to previously proposed algorithms, it is applicable in practice not only for regions where electrical properties are relatively constant but also for regions where they vary. The convective field of the convection-reaction equation depends on the spatial derivatives of the $B 1$ field, and in the regions where its magnitude is low, a spot-like artifact is observed in the reconstructed electrical properties images. For eliminating this artifact, two different methods are developed, namely "constrained cr-MREPT" and "double-excitation cr-MREPT." Successful reconstructions are obtained using noisy and noise-free simulated data, and experimental data from phantoms.
\end{abstract}

Index Terms-B1 mapping, conductivity imaging, convection-reaction equation, electrical impedance tomography (EIT), magnetic resonance electrical impedance tomography (MREIT), magnetic resonance electrical properties tomography (MREPT), permittivity imaging, quantitative magnetic resonance imaging (MRI), triangular mesh.

\section{INTRODUCTION}

I MAGING of electrical properties of tissues is useful for monitoring and diagnostic purposes [1]-[7]. It is known that electrical properties of tissues depend on frequency [5]. Electrical impedance tomography (EIT) and magnetic induction tomography (MIT) are developed to image electrical conductivity

Manuscript received October 10, 2013; revised December 16, 2013; accepted December 16, 2013. Date of publication January 02, 2014; date of current version February 27, 2014. This work was supported by The Scientific and Technological Research Council of Turkey (TUBITAK) under Grant 111E090. Asterisk indicates corresponding author.

F. S. Hafalir, O. F. Oran, and N. Gurler are with the Electrical and Electronics Engineering Department, Bilkent University, 06800 Ankara, Turkey.

*Y. Z. Ider is with the Electrical and Electronics Engineering Department, Bilkent University, 06800 Ankara, Turkey (e-mail: ider@ee.bilkent.edu.tr).

Color versions of one or more of the figures in this paper are available online at $\mathrm{http}: / /$ ieeexplore.ieee.org.

Digital Object Identifier 10.1109/TMI.2013.2296715 $(\sigma)$ and dielectric permittivity $(\varepsilon)$ of tissues in the frequency range $1 \mathrm{kHz}$ to $1 \mathrm{MHz}$ [8]-[13]. In these methods, current is either injected into the body by surface electrodes (EIT), or induced in the body using external coils (MIT), and data are measured either on the surface of the body or outside the body. Consequently, low spatial resolution is achieved especially for interior regions of the body because measured data are less sensitive to the variations of the electrical properties of such regions. In order to improve spatial resolution in the relatively interior regions, magnetic resonance electrical impedance tomography (MREIT) has been proposed [14]-[20]. In MREIT, internal magnetic field generated by the internal current distribution is imaged with high resolution using magnetic resonance imaging (MRI) techniques [21], [22]. Thereby local magnetic field perturbations due to local conductivity perturbations are sensitively measured resulting in higher spatial resolution also in the interior regions. For an internal ac current to accumulate phase, a refocusing radio-frequency (RF) pulse must be applied after each half-cycle of the current and thus currently MREIT is suitable for dc or up to $1 \mathrm{kHz}$ imaging of conductivity [23].

In addition to electrical properties of tissues at the frequencies mentioned above, their electrical properties at RF frequencies are also of interest especially for estimating specific absorption rate distributions during an MR experiment as well as for monitoring and diagnostic purposes. Several electrical property imaging techniques have been developed for the RF frequencies used in high field MR systems such as $1.5 \mathrm{~T}$ or higher and these are in general called magnetic resonance electrical properties tomography (MREPT) [24]-[35]. These techniques exploit the fact that the electrical properties of the imaged object perturb the RF magnetic field of the MRI system. Thus if the RF magnetic field is measured, then it may be possible to reconstruct the electrical properties. This study is confined to the reconstruction of tissue conductivity and dielectric permittivity or equivalently admittivity defined as $\gamma=\sigma+i \omega \varepsilon$, where $\omega$ is the frequency of the applied RF field. Imaging of magnetic permeability is not considered, and it is assumed that tissues have the free space magnetic permeability $\mu_{0}$.

Haacke et al. has suggested to calculate the conductivity and permittivity via the formula $\gamma=\left(\nabla^{2} B_{1}^{+}\right) /\left(i \omega \mu_{0} B_{1}^{+}\right)$where $B_{1}^{+}$is the MR-wise active circularly polarized (left-handed rotating) component of the RF field [24]. This algorithm, which we hereafter call the "std-MREPT method," is valid only in regions where electrical properties vary slowly. The first application of the std-MREPT method is described by Wen [25]. In this 
application, $B_{1}^{+}$magnitude map is found using the well-known double-angle $B_{1}$ mapping technique [36] and $B_{1}^{+}$phase distribution is assumed to be half of the spin-echo MR phase image in case a quadrature birdcage coil is used. This assumption on $B_{1}^{+}$ phase is called "transceive phase approximation (TPA)." The std-MREPT formula is a pointwise formula where the $\gamma$ value at a spatial location is determined from the ratio of $\nabla^{2} B_{1}^{+}$and $B_{1}^{+}$values obtained for that spatial location. Katscher et al. proposed a more robust algorithm in which in effect the ratio of suitable surface integrals of $\nabla^{2} B_{1}^{+}$and $B_{1}^{+}$around the point of interest is used [26]. Later, Voigt et al. proposed an algorithm which is derived by appropriate volume integrations of the numerator and the denominator of the std-MREPT equation [29]. These two methods do not require the explicit calculation of the second spatial derivatives of $B_{1}^{+}$, and they are effectively implementations of local averages of the std-MREPT equation. The algorithms of Katscher et al. and Voigt et al. still depend on the TPA. Furthermore, these algorithms are also only suitable for reconstructing conductivity and permittivity in regions where it may be assumed that these properties are constant or vary slowly. Errors incurring due to "nonconstant admittivity" are analyzed thoroughly in [37].

Zhang et al. have developed a "dual-excitation algorithm" whereby data are collected for two different linear RF excitations [27]. A total of four equations are derived to model these data in which admittivity, $\gamma$, and its three spatial derivatives appear as the unknown variables. By solving these equations, $\gamma$ is reconstructed and the artifacts which appear in nonconstant admittivity regions are significantly reduced. These investigators assume that the $B_{x}$ and $B_{y}$ components of the excitation RF field can be measured, and therefore this method is not easily applicable to most clinical MRI scanners at present [27]. (Note that z-direction is taken as the direction of the dc magnetic field of an MRI system). Alternatively, Nachman et al. derived an exact formula of admittivity as $\gamma=\left(\nabla^{2} \mathbf{H} \cdot(\nabla \times \mathbf{H})\right) /\left(i \omega \mu_{0} \mathbf{H} \cdot(\nabla \times\right.$ $\mathbf{H})$ ) [33]. However, this equation requires all spatial components of $\mathbf{H}$ to be measured and also in some regions, $\mathbf{H} \cdot(\nabla \times \mathbf{H})$ is small since $\mathbf{H}$ and $\nabla \times \mathbf{H}$ are almost orthogonal. Zhang and Nachman methods are still to be tested against real experimental data.

Sodickson et al. have recently proposed a method called "local Maxwell tomography" (LMT) and its extended version called "generalized local Maxwell tomography" [34], [35]. These methods are free of TPA and solve for unknown electrical properties using complementary information from the transmit and receive sensitivity distributions of multiple coils. LMT is only suitable for regions where electrical properties vary slowly whereas generalized LMT is suitable for all regions including the regions where the electrical properties have high gradients. However, the generalized LMT involves higher derivatives of the magnetic fields and therefore still needs to be assessed under realistic conditions including noisy data. Recent studies by Katscher et al. and Zhang et al. also make use of multi-channel data but still make the assumption of slow variation of electrical properties [30], [31].

In this study, we have proposed a novel algorithm called convection-reaction equation based MREPT (cr-MREPT) which is suitable for reconstructing electrical properties not only in re- gions where they are relatively constant but also in transition regions where they vary such as at the boundaries of internal objects. Starting from the Maxwell's equations, we derive a partial differential equation for admittivity, $\gamma$, which is in the form of the convection-reaction equation where the coefficients of the equation depend on the complex $B_{1}^{+}$map. The derived equation is then solved using a triangular mesh based finite difference method to reconstruct $\gamma$. Reconstructions are made using noise-free and noisy simulated data and also from experimental data.

\section{THEORY}

The RF magnetic field, $\mathbf{H}$, inside the object to be imaged, is primarily determined by the geometry of the RF coil and it is also influenced by the presence (loading effect) of the object. The loading effect of the object is related to its electrical properties, and specifically to its admittivity which is defined as $\gamma=\sigma+i \omega \varepsilon$ where $\sigma$ is electrical conductivity and $\varepsilon$ is dielectric permittivity. Although the influence of $\gamma$ on $\mathbf{H}$ is not desired in conventional imaging because it distorts the homogeneity of the RF field within the object, in MREPT, this influence is exploited. The purpose of this section is therefore to relate the perturbation in $\mathbf{H}$ to the admittivity distribution of the object, so that if $\mathbf{H}$ can be measured then an inverse problem may be solved to find admittivity.

Components of $\mathbf{H}$ can be expressed in terms of the left-handed rotating and right-handed rotating $\mathrm{RF}$ fields $H^{+}$, and $H^{-}$respectively, as $\mathbf{H}=\left(H_{x}, H_{y}, H_{z}\right)=$ $\left(H^{+}+H^{-*},-i H^{+}+i H^{-*}, H_{z}\right)$ where $H^{+}=\left(H_{x}+i H_{y}\right) / 2$, and $H^{-}=\left(H_{x}-i H_{y}\right)^{*} / 2$ [38]. It is assumed that $H^{+}$can be measured by MRI techniques and therefore in the forthcoming a relation between $H^{+}$and $\gamma$ is obtained.

Admittivity appears in Ampere's Law (with Maxwell's correction) as $\nabla \times \mathbf{H}=\gamma \mathbf{E}$. After taking the curl of both sides of this equation, by using the vector identity $\nabla \times \nabla \times \mathbf{H}=$ $-\nabla^{2} \mathbf{H}+\nabla \nabla \cdot \mathbf{H}$ and the fact that $\nabla \cdot \mathbf{H}=0$, and also by making use of the Faraday's Law $\nabla \times \mathbf{E}=-i \omega \mu_{0} \mathbf{H}$, we can obtain an equation involving the magnetic field only, as follows:

$$
\begin{aligned}
\nabla \times \nabla \times \mathbf{H} & =\nabla \times(\gamma \mathbf{E})=\nabla \gamma \times \mathbf{E}+\gamma \nabla \times \mathbf{E} \\
\Rightarrow-\nabla^{2} \mathbf{H} & =\frac{\nabla \gamma}{\gamma} \times(\nabla \times \mathbf{H})-i \omega \mu_{0} \gamma \mathbf{H} .
\end{aligned}
$$

We can write the $\mathrm{x}$ - and $\mathrm{y}$-components of (2) as

$$
\begin{aligned}
-\nabla^{2} H_{\mathrm{x}}= & \frac{1}{\gamma} \frac{\partial \gamma}{\partial y}\left(\frac{\partial H_{\mathrm{y}}}{\partial \mathrm{x}}-\frac{\partial H_{\mathrm{x}}}{\partial \mathrm{y}}\right) \\
& -\frac{1}{\gamma} \frac{\partial \gamma}{\partial z}\left(\frac{\partial H_{\mathrm{x}}}{\partial \mathrm{z}}-\frac{\partial H_{\mathrm{z}}}{\partial \mathrm{x}}\right)-i \omega \mu_{0} \gamma H_{x} \\
-\nabla^{2} H_{\mathrm{y}}= & \frac{1}{\gamma} \frac{\partial \gamma}{\partial z}\left(\frac{\partial H_{\mathrm{z}}}{\partial \mathrm{y}}-\frac{\partial H_{\mathrm{y}}}{\partial \mathrm{z}}\right) \\
& -\frac{1}{\gamma} \frac{\partial \gamma}{\partial x}\left(\frac{\partial H_{\mathrm{y}}}{\partial \mathrm{x}}-\frac{\partial H_{\mathrm{x}}}{\partial \mathrm{y}}\right)-i w \mu_{0} \gamma H_{y} .
\end{aligned}
$$


If we multiply (4) by $i$ and add to (3), we obtain

$$
\begin{array}{r}
-2 \nabla^{2} H^{+}=-\frac{1}{\gamma} \frac{\partial \gamma}{\partial x} i\left(\frac{\partial H_{y}}{\partial x}-\frac{\partial H_{x}}{\partial y}\right)-\frac{1}{\gamma} \frac{\partial \gamma}{\partial y}\left(-\frac{\partial H_{y}}{\partial x}+\frac{\partial H_{x}}{\partial y}\right) \\
-\frac{1}{\gamma} \frac{\partial \gamma}{\partial z}\left[2 \frac{\partial H^{+}}{\partial z}-\frac{\partial H_{z}}{\partial x}-i \frac{\partial H_{z}}{\partial y}\right]-2 i \omega \mu_{0} \gamma H^{+} .
\end{array}
$$

By using the definition of $H^{+}$and the fact that $\nabla \cdot \mathbf{H}=$ $\left(\partial H_{x} / \partial x\right)+\left(\partial H_{y} / \partial y\right)+\left(\partial H_{z} / \partial z\right)=0$ we can modify the $\left(\left(\partial H_{\mathrm{y}} / \partial \mathrm{x}\right)-\left(\partial H_{\mathrm{x}} / \partial \mathrm{y}\right)\right)$ factor as

$$
\begin{aligned}
\frac{\partial H_{\mathrm{y}}}{\partial \mathrm{x}}-\frac{\partial H_{\mathrm{x}}}{\partial \mathrm{y}} & =\frac{\partial H_{\mathrm{y}}}{\partial \mathrm{x}}-\frac{\partial H_{\mathrm{x}}}{\partial \mathrm{y}}-i\left(\frac{\partial H_{x}}{\partial x}+\frac{\partial H_{y}}{\partial y}+\frac{\partial H_{z}}{\partial \mathrm{z}}\right) \\
& =-2 i\left(\frac{\partial H^{+}}{\partial x}-i \frac{\partial H^{+}}{\partial y}+\frac{1}{2} \frac{\partial H_{z}}{\partial \mathrm{z}}\right) .
\end{aligned}
$$

By using this identity, (5) becomes

$$
\begin{aligned}
-\nabla^{2} H^{+}= & -\frac{1}{\gamma} \frac{\partial \gamma}{\partial x}\left(\left(\frac{\partial H^{+}}{\partial x}-i \frac{\partial H^{+}}{\partial y}\right)+\frac{1}{2} \frac{\partial H_{z}}{\partial z}\right) \\
& -\frac{1}{\gamma} \frac{\partial \gamma}{\partial y}\left(i\left(\frac{\partial H^{+}}{\partial x}-i \frac{\partial H^{+}}{\partial y}\right)+\frac{i}{2} \frac{\partial H_{z}}{\partial z}\right) \\
& -\frac{1}{\gamma} \frac{\partial \gamma}{\partial z}\left[\frac{\partial H^{+}}{\partial z}-\frac{1}{2} \frac{\partial H_{z}}{\partial x}-\frac{i}{2} \frac{\partial H_{z}}{\partial y}\right]-i \omega \mu_{0} \gamma H^{+}
\end{aligned}
$$

By dividing by $\gamma$ and using the definition $u=1 / \gamma$, (6) can be written as

$\boldsymbol{\beta} \cdot \nabla u+\nabla^{2} H^{+} u-i \omega \mu_{0} H^{+}=0 \quad$ where

$\nabla u=\left[\begin{array}{l}\frac{\partial u}{\partial x} \\ \frac{\partial u}{\partial y} \\ \frac{\partial u}{\partial z}\end{array}\right]=\left[\begin{array}{c}-\frac{1}{\gamma^{2}} \frac{\partial \gamma}{\partial x} \\ -\frac{1}{\gamma^{2}} \frac{\partial \gamma}{\partial y} \\ -\frac{1}{\gamma^{2}} \frac{\partial \gamma}{\partial z}\end{array}\right], \boldsymbol{\beta}=\left[\begin{array}{c}\beta_{x} \\ \beta_{y} \\ \beta_{z}\end{array}\right]=\left[\begin{array}{l}\frac{\partial H^{+}}{\partial x}-i \frac{\partial H^{+}}{\partial y}+\frac{1}{2} \frac{\partial H_{z}}{\partial z} \\ i \frac{\partial H^{+}}{\partial x}-\frac{\partial H^{+}}{\partial y}+\frac{i}{2} \frac{\partial H_{z}}{\partial z} \\ \frac{\partial H^{+}}{\partial z}-\frac{1}{2} \frac{\partial H_{z}}{\partial x}-\frac{i}{2} \frac{\partial H_{z}}{\partial y}\end{array}\right]$

This equation is the well-known convection-diffusion-reaction equation with null diffusion term, where $\beta$ is the convective field and $\nabla^{2} H^{+} u-i \omega \mu H^{+}$is the reaction component. (Note that $\beta_{y}=i \beta_{x}$ )

We have already assumed that $H^{+}$can be measured using MRI. If additionally the gradient of $H_{z}$ is known, then (7) can be solved in three dimensions for $u$ by imposing appropriate boundary conditions. However, measurement of $H_{z}$ component is not feasible in MRI. On the other hand, $H_{z}$ can be neglected in the central regions for a birdcage RF coil where end-ring generated $H_{z}$ field is minimum. In many reconstruction applications, $u$ is desired to be found in a specified xy-plane (slice). For such applications, if it can be assumed that $\partial u / \partial z$ is negligible for the slice of interest then (7) can be simplified into its 2-D form which is

$$
\begin{aligned}
& \mathbf{F} \cdot \bar{\nabla} u+\nabla^{2} H^{+} u-i \omega \mu_{0} H^{+}=0 \\
& \text { where } \bar{\nabla} u=\left[\begin{array}{c}
\frac{\partial u}{\partial x} \\
\frac{\partial u}{\partial y}
\end{array}\right] \text { and } \mathbf{F}=\left[\begin{array}{c}
F_{x} \\
F_{y}
\end{array}\right]=\left[\begin{array}{c}
\frac{\partial H^{+}}{\partial x}-i \frac{\partial H^{+}}{\partial y} \\
i \frac{\partial H^{+}}{\partial x}+\frac{\partial H^{+}}{\partial y}
\end{array}\right] .
\end{aligned}
$$

If $\bar{\nabla} u$ is assumed to be negligible such as in regions where electrical properties vary slowly, then the solution of (8) reduces to

$$
u=\frac{i \omega \mu_{0} H^{+}}{\nabla^{2} H^{+}} .
$$

This formula is in effect the same as the Haacke's formula mentioned in the introduction except that $u=1 / \gamma$. (Note that the symbol $\mathrm{H}^{+}$used in this section and the symbol $B_{1}^{+}$used frequently in the literature both represent the left-handed rotating RF field and that $B_{1}^{+}=\mu_{0} H^{+}$).

The coefficients of the partial differential equation in (8) depend on $\mathrm{H}^{+}$and therefore $\mathrm{H}^{+}$must be measured. Measurement of the magnitude and phase of $H^{+}$is explained in Section III-C2.

\section{METHODS}

\section{A. Solution of the Convection-Reaction Equation of MREPT (cr-MREPT)}

In this study, in order to reconstruct $\sigma$ and $\varepsilon,(8)$ is solved for $u$. A triangular mesh based finite difference method is proposed where a triangular mesh is generated in the imaging slice as a first step. It is assumed that $H^{+}$is measured (known) on the nodes of the triangular mesh. The procedure for obtaining $\mathrm{H}^{+}$ distribution on the nodes from the MR raw data is discussed in the Section III-C. Equation (8), which is a partial differential equation, has the first derivatives and the Laplacian of $\mathrm{H}^{+}$as its space dependent coefficients. In the following, it is assumed that these coefficients are already calculated on the nodes (the procedure for calculating these first derivatives and the Laplacian is discussed at the end of this section).

Inside each triangular element, $u$ can be approximated as

$$
u(x, y)=\sum_{i=1}^{3} u_{i, j} \phi_{i, j}(x, y) \quad(x, y) \in \Omega_{j}, j=1,2, \ldots \mathrm{N}_{t}
$$

where $\Omega_{j}$ denotes the inside of the $j$ th triangle, $\mathrm{N}_{t}$ is the number of triangles in the imaging slice, $u_{i, j}$ is the value of $u$ at the $i$ th node of the $j$ th triangle, and $\phi_{i, j}(x, y)=a_{i, j} x+b_{i, j} y+$ $c$. In the finite element method (FEM) literature, $\phi_{i, j}(x, y)$ is called "linear shape function." The coefficients, $a, b$, and $c$, in these equations can be calculated by using the definitions $\phi_{i, j}\left(x_{m, j}, y_{m, j}\right)=1$ if $i=m$ and $\phi_{i, j}\left(x_{m, j}, y_{m, j}\right)=0$ otherwise where $\left(x_{m, j}, y_{m, j}\right)$ are the coordinates of the $m$ th node of the $j$ th triangle $(i=1,2,3$, and $m=1,2,3)$. Once the coefficients are determined, $\partial u / \partial x$ and $\partial u / \partial y$ are found inside the $j$ th triangle as follows:

$$
\frac{\partial u(x, y)}{\partial x}=\sum_{i=1}^{3} u_{i, j} a_{i, j} \text { and } \frac{\partial u(x, y)}{\partial y}=\sum_{i=1}^{3} u_{i, j} b_{i, j} .
$$

Similar to how $u$ is approximated in (10), each of $F_{x}, F_{y}$ and $\nabla^{2} H^{+}$can also be approximated in a triangle using their nodal 
values and the linear shape functions. Using these approximations and also (11), (8) can be written for each triangle as

$$
\begin{aligned}
& \sum_{i=1}^{3} F_{i, j}^{x} \phi_{i, j}(x, y) \sum_{i, 1}^{3} u_{i, j} a_{i, j}+\sum_{i=1}^{3} F_{i, j}^{y} \phi_{i, j}(x, y) \\
& \quad \times \sum_{i=1}^{3} u_{i, j} b_{i, j}+\sum_{i=1}^{3} \nabla^{2} H_{i, j}^{+} \phi_{i, j}(x, y) \sum_{i=1}^{3} u_{i, j} \phi_{i, j}(x, y) \\
& \quad=i \omega \mu_{0} \sum_{i=1}^{3} H_{i, j}^{+} \phi_{i, j}(x, y)
\end{aligned}
$$

where $F_{i, j}^{x}, F_{i, j}^{y}, \nabla^{2} H_{i, j}^{+}$, and $H_{i, j}^{+}$are $F_{x}, F_{y}, \nabla^{2} H^{+}$and $H^{+}$ values at the $i$ th node of the $j$ th triangle, respectively (please note that the $i$ in the factor $i \omega \mu_{0}$ is the unit imaginary number). Evaluating (12) at the centroid of the $j$ th triangle, denoted by $\left(x_{j}, y_{j}\right)$, and rearranging terms, one obtains

$$
\sum_{i=1}^{3} u_{i, j}\left(a_{i, j} F_{j}^{x}+b_{i, j} F_{j}^{y}+\nabla^{2} H_{j}^{+}\right)=i \omega \mu_{0} H_{j}^{+}
$$

where $F_{j}^{x}, F_{j}^{y}, \nabla^{2} H_{j}^{+}$, and $H_{j}^{+}$are defined at the centroid of the $j$ th triangle and they are the means of the three corresponding nodal values (note that $F_{j}^{x}=\sum_{i=1}^{3} F_{i, j}^{x} \phi_{i, j}\left(x_{j}, y_{j}\right)=$ $\sum_{i=1}^{3} F_{i, j}^{x} / 3$ and similarly for $F_{j}^{y}, \nabla^{2} H_{j}^{+}$, and $H_{j}^{+}$). Assigning global indexes to all nodes in the imaging slice, (13) is written for the $j$ th triangle as

$$
\sum_{k} u_{k}\left(a_{k} F_{j}^{x}+b_{k} F_{j}^{y}+\nabla^{2} H_{j}^{+}\right)=i \omega \mu_{0} H_{j}^{+}
$$

where $k \in P_{j}$ and $P_{j}$ by definition contains three integers which are the global indexes of the nodes of the $j$ th triangle. Equation (14) can be written for each triangle and a matrix system is obtained as

$$
\mathbf{K}_{\mathrm{N}_{t} \times \mathrm{N}_{p}} \mathbf{u}_{\mathrm{N}_{p} \times 1}=\mathbf{f}_{\mathrm{N}_{t} \times 1}
$$

where $\mathrm{N}_{t}$ is the number of triangles and $\mathrm{N}_{p}$ is the number of nodes on the imaging slice. Note that each row of the $\mathbf{K}$ matrix has only three nonzero elements. For the solution of (8), boundary conditions should also be considered. In this study, $u$ values at the boundary are assumed to be known (Dirichlet boundary condition). This information is used to eliminate corresponding columns of the matrix $\mathbf{K}$ and the number of unknowns $(\mathrm{Np})$ is decreased. Since $\mathrm{N}_{t}>\mathrm{N}_{p}$, the system is overdetermined and it is solved in the least-square sense.

As discussed in Section IV-A, in some cases, it is desired to specify $u$ values in a certain region and use this information as a priori knowledge (as a constraint). The $u$ values in this region are calculated beforehand whether using another reconstruction method or they are assumed to be known. Similar to the boundary nodes, this information is incorporated in the solution by eliminating corresponding columns of the matrix $\mathbf{K}$ and the number of unknowns $\left(\mathrm{N}_{p}\right)$ is further decreased.

As discussed above, $\mathbf{K}$ matrix and $\mathbf{f}$ vector in (15) are constructed using the measured $H^{+}$data and thus they are strictly related to the distribution of $\mathrm{H}^{+}$. Therefore, it is possible to obtain different $\mathbf{K}$ and $\mathbf{f}$ for different $\mathrm{RF}$ excitations which have

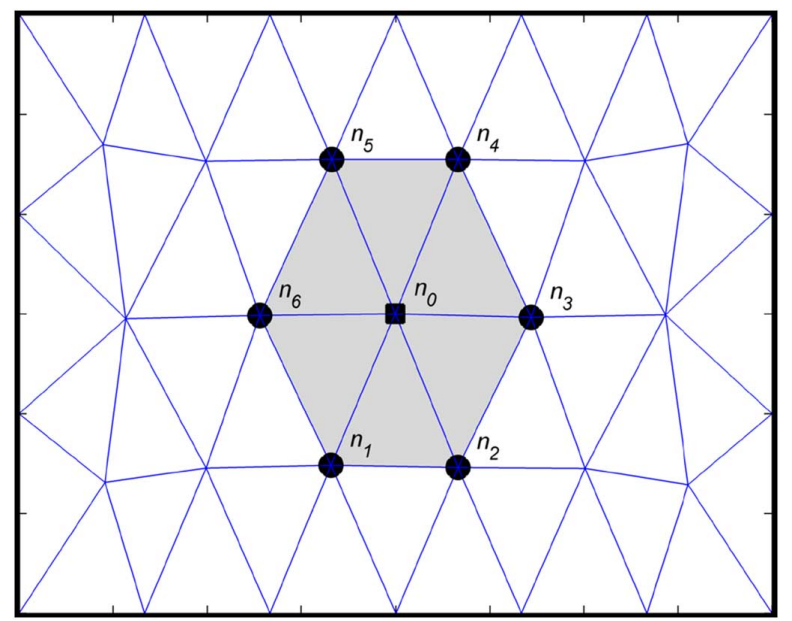

Fig. 1. Sample region of the triangular mesh at the imaging slice: $\mathbf{n}_{0}$ and its six neighboring nodes $\left(\mathrm{n}_{1}\right.$ to $\left.\mathrm{n}_{6}\right)$ are shown. $H^{+}$is approximated as a second-order polynomial in the shaded region using the $H^{+}$values at the nodes $n_{0}$ to $n_{6}$.

different $H^{+}$distributions. Let $\mathbf{K}_{\mathbf{1}}, \mathbf{K}_{\mathbf{2}}$ and $\mathbf{f}_{\mathbf{1}}, \mathbf{f}_{\mathbf{2}}$ be obtained for two different RF excitations. In this case, these matrices and vectors can be concatenated for the solution of $u$ as follows:

$$
\left[\begin{array}{l}
\mathbf{K}_{\mathbf{1}} \\
\mathbf{K}_{\mathbf{2}}
\end{array}\right]_{2 \mathrm{~N}_{t} \times \mathrm{N} p} \mathbf{u}_{\mathrm{N}_{p} \times 1}=\left[\begin{array}{l}
\mathbf{f}_{\mathbf{1}} \\
\mathbf{f}_{\mathbf{2}}
\end{array}\right]_{2 \mathrm{~N}_{t} \times 1} .
$$

Similar to the case when (15) is solved alone, the boundary conditions and the a priori knowledge (if desired) are used to eliminate corresponding columns of the concatenated matrix in (16) and the number of unknowns is decreased. The final matrix system is also over-determined and it is solved in the least-square sense. In Section IV-A, the rationale behind using two RF excitations resulting in two different $H^{+}$distributions rather than a single excitation is discussed.

1) Calculation of the First Derivatives and the Laplacian of $\mathrm{H}^{+}$at the Triangular Mesh Nodes: For the calculation of the first derivatives and the Laplacian of $\mathrm{H}^{+}$at the mesh nodes the method proposed by Fernandez et al. is used [39]. This method is in fact the implementation of Savitzky-Golay filtering on a triangular mesh [40]. It is assumed that $H^{+}$is known at the nodes of the triangular mesh defined in the imaging slice. Using nodal $\mathrm{H}^{+}$values, the first derivatives and the Laplacian of $\mathrm{H}^{+}$ are calculated separately for every node as follows: Let $n_{0}$ denote the node where the first derivatives and the Laplacian of $H^{+}$are to be calculated and let $n_{1}$ to $n_{6}$ denote the neighboring nodes of the central node $n_{0}$, as shown in Fig. $1 . H^{+}$is approximated as a second-order polynomial in the shaded region as $H^{+}(x, y)=c_{1} x^{2}+c_{2} y^{2}+c_{3} x y+c_{4} x+c_{5} y+c_{6}$. To find the coefficients, $c_{1}, c_{2}, \ldots, c_{6}$ the following set of equations is written:

$$
\begin{aligned}
& H^{+}(x, y)=c_{1} x_{i}^{2}+c_{2} y_{i}^{2} \\
& \quad+c_{3} x_{i} y_{i}+c_{4} x_{i}+c_{5} y_{i}+c_{6} \quad \text { for } i=0,1, \ldots, 6
\end{aligned}
$$

where $x_{i}$ and $y_{i}$ are the $x$-and $y$ - coordinates of node $i$. Note that in this system there are six unknowns and seven equations, and therefore the system is solved in the least square sense. However, for some nodes, such as the boundary nodes, the number 
of equations is less than 6 . In such a case, the minimum-norm solution is used for finding the coefficients. Once the coefficients of the second-order polynomial are determined, the first derivatives and the 2-D Laplacian of $H^{+}$for node $n_{0}$ are found as

$$
\begin{aligned}
& \frac{\partial H^{+}}{\partial x}=2 c_{1} x_{0}+c_{3} y_{0}+c_{4}, \\
& \frac{\partial H^{+}}{\partial y}=2 c_{2} y_{0}+c_{3} x_{0}+c_{5}, \text { and } \bar{\nabla}^{2} H^{+}=2 c_{1}+2 c_{2}
\end{aligned}
$$

where $x_{0}$ and $y_{0}$ are the $x$ - and $y$-coordinates of node $n_{0}$. This procedure is repeated for every node of the triangular mesh.

It should be noted that $\nabla^{2} H^{+}$also involves the second derivative of $H^{+}$with respect to $\mathrm{z}$. In simulations, $H^{+}$is calculated on two other slices one $5 \mathrm{~mm}$ above the imaging slice and one $5 \mathrm{~mm}$ below the imaging slice. In the experiments, $\mathrm{H}^{+}$is measured also on three slices with $5 \mathrm{~mm}$ spacing. Therefore, the second derivative of with respect to $\mathrm{z}$ is calculated using central difference approximation.

\section{B. Simulation Methods}

To test the proposed algorithms, simulated data are obtained using MATLAB (The Mathworks, Natick, MA, USA), and COMSOL Multiphysics 4.2a (COMSOL AB, Stockholm, Sweden), a FEM-based software package. MATLAB and COMSOL Multiphysics are also used for the implementation of reconstruction algorithms, filters, preprocessing steps, and all numerical procedures.

In the simulations, 24-leg high-pass shielded birdcage coil with a radius of $30 \mathrm{~cm}$ and length of $1 \mathrm{~m}$ shown in Fig. 2 is modeled in COMSOL Multiphysics [41]. In order to generate a homogenous $\mathrm{H}^{+}$in the region of interest (ROI) for the unloaded case, optimum capacitance value is calculated as $8.6 \mathrm{pF}$ at $123.2 \mathrm{MHz}$ (corresponding to the 2.89T MRI system used in this study) using the method proposed in [42]. The variation of $\left|H^{+}\right|$is less than $\pm 2 \%$ within a cylindrical region of $30 \mathrm{~cm}$ length along the z-axis and $15 \mathrm{~cm}$ in radius. Quadrature excitation is used in transmit mode during which the birdcage coil is driven by $500 \mathrm{~V}$ peak from two ports which are geometrically $90^{\circ}$ apart from each other and with $90^{\circ}$ phase difference. In some cases such as in Section IV-A3, when we need to calculate the phase due to the receive sensitivity distribution, we switch the ports and calculate $H^{-}$.

1) Simulation Phantoms: As the loading objects, three different phantoms shown in Fig. 3 are modeled in the simulation environment. These simulation phantoms are cylindrical in nature with outer diameter of $14.4 \mathrm{~cm}$ and height of $19.5 \mathrm{~cm}$.

The "first simulation phantom" consists of two regions, A and B. Region A has a diameter of $7.5 \mathrm{~cm}$.

The "second simulation phantom," on the other hand, consists of three regions, $\mathrm{C}, \mathrm{D}$, and $\mathrm{E}$. The region $\mathrm{C}$ has a diameter of $5 \mathrm{~cm}$. For this phantom, electromagnetic simulations are made with and without region $\mathrm{E}$ (i.e., region $\mathrm{E}$ is cut out when so desired) and thus two different $\mathrm{H}^{+}$simulated data in the ROI are acquired. For this phantom the ROI is defined as the union of regions $\mathrm{C}$ and $\mathrm{D}$.

The "third simulation phantom," which is more complex than the others, consists of five regions $\left(\mathrm{F}_{1}, \mathrm{~F}_{2}, \mathrm{~F}_{3}, \mathrm{G}\right.$, and $\left.\mathrm{H}\right)$. The

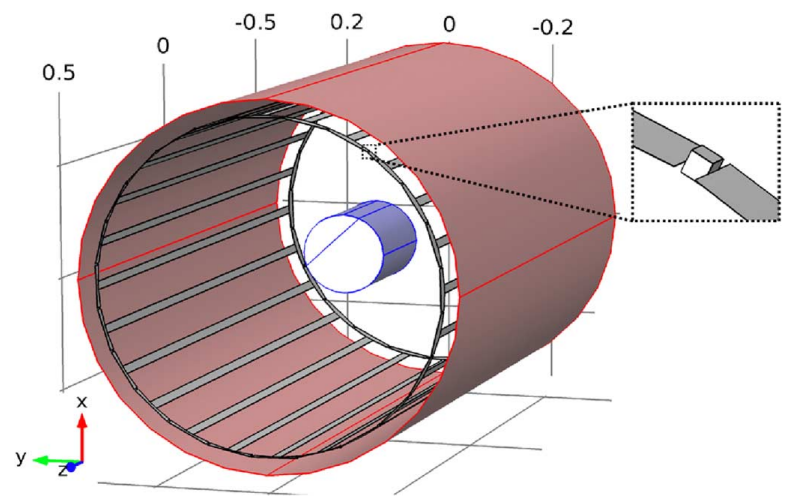

Fig. 2. Geometry of the 24-leg shielded high-pass birdcage coil with a radius of $30 \mathrm{~cm}$ and length of $1 \mathrm{~m}$ and the cylindrical phantom inside the coil. The inset shows the magnified view of a capacitor. For driving the birdcage coil one or more capacitors are used as ports by applying voltage across the capacitor plates.

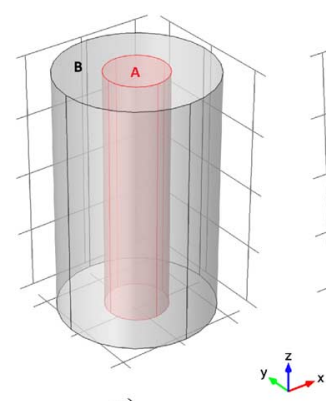

a)

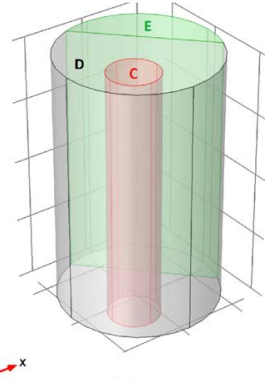

b)

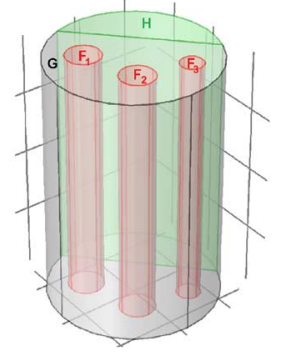

c)
Fig. 3. (a) First, (b) the second, and (c) the third simulation phantoms.

cylindrical regions, $\mathrm{F}_{1}, \mathrm{~F}_{2}$, and $\mathrm{F}_{3}$, have radii of $1.5,1.5$, and $1 \mathrm{~cm}$. For this phantom also two electromagnetic simulations are made. In one of the simulations regions $\mathrm{G}$ and $\mathrm{H}$ have the same; and in the other simulation they have different electrical properties. In this phantom the ROI is defined as the union of $\mathrm{F}_{1}, \mathrm{~F}_{2}, \mathrm{~F}_{3}$, and $\mathrm{G}$.

In general, one can perceive regions $\mathrm{B}, \mathrm{D}$, and $\mathrm{G}$ as the background; and regions $\mathrm{A}, \mathrm{C}, \mathrm{F}_{1}, \mathrm{~F}_{2}$, and $\mathrm{F}_{3}$ as the anomaly objects. Regions $\mathrm{E}$ and $\mathrm{H}$ are the "padding" regions. The concept of "padding" is explained in Section IV-A. The actual conductivity and permittivity values of different regions of the simulation phantoms are as follows: For regions A and C, $\sigma=1 \mathrm{~S} / \mathrm{m}$ and $\varepsilon_{r}=50$; for regions $\mathrm{B}, \mathrm{D}$, and $\mathrm{E}, \sigma=0.5 \mathrm{~S} / \mathrm{m}$ and $\varepsilon_{r}=75$; for regions $\mathrm{F}_{1}, \mathrm{~F}_{2}, \mathrm{~F}_{3}$, and $\mathrm{H} \sigma=1.25 \mathrm{~S} / \mathrm{m}$ and $\varepsilon_{r}=76$; for region $\mathrm{G} \sigma=0.6 \mathrm{~S} / \mathrm{m}$ and $\varepsilon=77.2$. It should be noted that in all three simulation phantoms material properties do not change in the z-direction.

2) Obtaining Noisy Simulated Data: In order to obtain noisy complex $\mathrm{H}^{+}$data, the following procedure is applied.

a) Obtaining noisy $\mathrm{H}^{+}$magnitude.

i) Simulated $\left|H^{+}\right|$is obtained.

ii) MR magnitude image with nominal $60^{\circ}$ flip angle is obtained using the formula $S_{1}=\sin \left(k\left|H^{+}\right|\right)$. The constant $k$ is determined so that the average flip angle in the imaging slice is $60^{\circ}$. MR magnitude image with nominal $120^{\circ}$ flip angle is obtained by the formula $\left.S_{2}=\sin \left(2 k\left|H^{+}\right|\right)\right)$. 
iii) An SNR value for MR magnitude image is assumed.

iv) Gaussian white noise is added to $S_{1}$ with standard deviation $s d=A / S N R$ where $A$ is the mean of $S_{1}$ magnitude image. Another Gaussian white noise with the same standard deviation is added to $S_{2}$.

v) Using noisy $S_{1}$ and $S_{2}$ magnitude images, noisy $\mathrm{H}^{+}$magnitude is obtained using the double angle $B_{1}$ mapping formula, $\left|H^{+}\right|=\cos ^{-1}\left(S_{2} / 2 S_{1}\right) / k$.

b) Obtaining noisy $H^{+}$phase.

The noise in MRI phase images is assumed to have zero-mean Gaussian distribution with standard deviation $S d_{\Phi}=\sqrt{2} / S N R$ where SNR is the signal-to-noise ratio of the MRI magnitude image [43]. Since $H^{+}$phase is assumed to be half of the MRI spin-echo phase, the noise in $H^{+}$phase image becomes $s d_{\Phi_{B 1}}=1 /(\sqrt{2} \mathrm{SNR})$.

c) Obtaining noisy complex $\mathrm{H}^{+}$

Noisy complex $H^{+}$is obtained from the noisy $H^{+}$magnitude and phase, using Euler's formula.

In the simulations, SNR values of 50,100, and 150 are used. These SNR values are reasonable for regular MRI scanning. In fact, the SNRs of the MRI magnitude images obtained experimentally throughout this study were estimated to be in the range of 50-100. It has been shown that, for SNR values of greater than 3, the probability distributions of noise in both MRI magnitude and phase images are very close to Gaussian [43], [44]. A low pass filter with Gaussian kernel (in the spatial domain) with standard deviation $0.0032 \mathrm{~m}$ was applied to the noisy simulated $\mathrm{H}^{+}$complex images. The filter was applied using nonlinear diffusion based denoising technique [45].

Errors made in the reconstructed conductivity and permittivity images are calculated using the relative $L^{2}$-error formulae

$$
\begin{aligned}
& \mathrm{E}_{L^{2}}(\sigma)=100\left[\frac{\sum_{j=1}^{N_{p}}\left(\sigma_{j}^{a}-\sigma_{j}\right)^{2}}{\sum_{j=1}^{N_{p}}\left(\sigma_{j}^{a}\right)^{2}}\right]^{\frac{1}{2}} \\
& \mathrm{E}_{L^{2}}(\varepsilon)=100\left[\frac{\sum_{j=1}^{N_{p}}\left(\varepsilon_{j}^{a}-\varepsilon_{j}\right)^{2}}{\sum_{j=1}^{N_{p}}\left(\varepsilon_{j}^{a}\right)^{2}}\right]^{\frac{1}{2}}
\end{aligned}
$$

where $\sigma_{j}^{a}\left(\varepsilon_{j}^{a}\right)$ and $\sigma_{j}\left(\varepsilon_{j}\right)$ are the actual and reconstructed conductivity (permittivity) distributions at the $j$ th node, respectively.

\section{Experimental Methods}

In order to test the proposed MREPT algorithm with experimental data, three experimental setups are prepared. For these setups, the simulation phantoms shown in Fig. 3(a)-(c) are manufactured from plexiglass and they are called the first, second, and the third experimental phantoms.

1) Phantom Preparation: For the first experimental phantom, the background [region B in Fig. 3(a)] is made by using an agar-saline gel $(20 \mathrm{gr} / 1 \mathrm{Agar}, 2 \mathrm{gr} / \mathrm{l} \mathrm{NaCl}, 1.5$ $\left.\mathrm{gr} / \mathrm{l} \mathrm{CuSO}_{4}\right) . \mathrm{NaCl}$ is used for adjusting the conductivity of the phantom and $\mathrm{CuSO}_{4}$ is used for decreasing $\mathrm{T}_{1}$ of the solution to around $300 \mathrm{~ms}$. After region B is solidified (within several hours), region A [shown in Fig. 3(a)] is filled with a saline solution $\left(6 \mathrm{gr} / 1 \mathrm{NaCl}, 1.5 \mathrm{gr} / 1 \mathrm{CuSO}_{4}\right)$ of different conductivity in order to obtain conductivity contrast. Since $\mathrm{NaCl}$ diffusion between region $\mathrm{A}$ and $\mathrm{B}$ affects the conductivity distribution in the ROI, the data acquisition is started right after region A is filled. The total time it takes for data acquisition to be completed is $64 \mathrm{~min}$ as explained in the next section on "measurement of $H^{+}$."

The second experimental phantom is prepared by applying similar steps as above: Regions D and E in Fig. 3(b) are built using an agar-saline gel (20 gr/l Agar, $2 \mathrm{gr} / 1 \mathrm{NaCl}, 1.5$ $\left.\mathrm{gr} / \mathrm{l} \mathrm{CuSO}_{4}\right)$ and region $\mathrm{C}$ is filled with a saline solution of different conductivity $\left(6 \mathrm{gr} / 1 \mathrm{NaCl}, 1.5 \mathrm{gr} / 1 \mathrm{CuSO}_{4}\right)$. Immediately after region $\mathrm{C}$ is filled, a complete data acquisition is performed in $64 \mathrm{~min}$. At the end of this first acquisition the agar-saline region $\mathrm{E}$ is cut out, and a second acquisition is started. Thus, two different experiments, with and without region $\mathrm{E}$, are performed using this phantom in order to obtain different $H^{+}$distributions. These two experiments take about $2 \times 64=128 \mathrm{~min}$.

The third experimental phantom is similarly prepared: Region $\mathrm{G}$ in Fig. 3(c) is built using an agar-saline gel $(20 \mathrm{gr} / \mathrm{l}$ Agar, $3.4 \mathrm{gr} / 1 \mathrm{NaCl}, 1.5 \mathrm{gr} / \mathrm{C} \mathrm{CuSO}_{4}$ ) and regions $\mathrm{F}_{1}, \mathrm{~F}_{2}$, and $\mathrm{F}_{3}$ are filled with a saline solution of different conductivity $(7.4 \mathrm{gr} / 1$ $\left.\mathrm{NaCl}, 1.5 \mathrm{gr} / 1 \mathrm{CuSO}_{4}\right)$. Immediately following the filling of regions $\mathrm{F}_{1}, \mathrm{~F}_{2}$, and $\mathrm{F}_{3}$, data acquisition is started. With this phantom also two experiments are carried out. In the first experiment, region $\mathrm{H}$ is empty, and in the second experiment, region $\mathrm{H}$ is also filled with the same solution as used for regions $\mathrm{F}_{1}$, $\mathrm{F}_{2}$, and $\mathrm{F}_{3}$. Again the total time for the completion of the two experiments is $128 \mathrm{~min}$.

In preparing our saline solutions, the required $\mathrm{NaCl}$ concentration for target conductivity is calculated using the formula provided in [46]. For the first and second experimental phantoms the saline solution is expected to have conductivity of 1.0 $\mathrm{S} / \mathrm{m}$ due to $\mathrm{NaCl}$. It is also expected that the presence of $\mathrm{CuSO}_{4}$ will increase the conductivity of our saline solution by about $0.07 \mathrm{~S} / \mathrm{m}$ [47]. Indeed using a conductivity meter (Hanna Instruments, HI 8733) with four-ring potentiometric probe we have measured the conductivity to be $1.05 \mathrm{~S} / \mathrm{m}$. For the third experimental phantom, conductivity of the saline solution was measured to be $1.27 \mathrm{~S} / \mathrm{m}$ which is very close to the expected value of $1.29 \mathrm{~S} / \mathrm{m}$. The probe of the conductivity meter is not suitable for measurement in agar-saline gels. For agar-saline gels one must also consider the effect of agar to electrical conductivity. Agar may contribute an additional conductivity of up to 0.075 $\mathrm{S} / \mathrm{m}$ at $114 \mathrm{MHz}$ if $2 \%$ agar is used, as deduced from the work of Iizuka [48]. For agar parts of first and second experimental phantoms, we expect the conductivity to be $0.47 \mathrm{~S} / \mathrm{m}$ including $\mathrm{CuSO}_{4}$ and agar effects. For the third experimental phantom, this value is $0.66 \mathrm{~S} / \mathrm{m}$. It has been demonstrated that for saline solutions conductivity is fairly constant in the range $10 \mathrm{~Hz}$ to $1 \mathrm{GHz}$ [8], [49]. Therefore, the above estimates of conductivity are representative for the $123.2 \mathrm{MHz}$ Larmor frequency of the MRI system used in the study.

For the different $\mathrm{NaCl}$ concentrations that we have used in our experimental phantoms, the dielectric permittivity formula given in [46] for $\mathrm{NaCl}$ solutions indicates that the differences in dielectric permittivity are less than $\% 1$ and on the average their relative permittivity is about 77 which is very close the salt-free water relative permittivity of 80 . It is also stated in [46] that, in the frequency range $10 \mathrm{~Hz}$ to $200 \mathrm{MHz}$, dielectric permittivity 
of $\mathrm{NaCl}$ solutions does not change. Similarly, Iizuka [48] shows that agar effect on permittivity is also negligible at $114 \mathrm{MHz}$. We therefore conclude that in our experimental phantoms dielectric permittivity does not change significantly across their cross section.

Hamamura et al. have studied the diffusion of $\mathrm{NaCl}$ in Agar phantoms prepared for MREIT experiments [50]. They have found that for a $20 \mathrm{gr} / 1$ Agar preparation the diffusion constant is in the order of $10^{-9} \mathrm{~m}^{2} / \mathrm{s}$. Solving the diffusion equation for the first experimental phantom using Comsol Multiphysics we have found that the $10 \%-90 \%$ rise length of $\mathrm{NaCl}$ concentration at the boundary of the internal cylinder becomes $4,6,8,12 \mathrm{~mm}$ after a waiting period of $0.5,1,2,4 \mathrm{~h}$, respectively. Similar widening of originally sharp conductivity boundaries would be expected in view of the fact that conductivity is approximately proportional to $\mathrm{NaCl}$ concentration. Therefore in our experiment, given that the total data acquisition takes $1-2 \mathrm{~h}$, widening of boundaries in the range $6-8 \mathrm{~mm}$ are expected, which is not critical in view of the fact that the data is noisy and we use a low-pass filter before reconstruction.

2) Measurement of $\mathrm{H}^{+}$: Magnitude of $\mathrm{H}^{+}$can be found by one of the several available $B_{1}$ mapping techniques [36], [51]-[54]. In this study we have used the double-angle-method [36]: Two MR magnitude images, $\left|M_{1}\right|$ and $\left|M_{2}\right|$, are acquired by using two gradient-echo pulse sequences of nominal flip angles $60^{\circ}$ and $120^{\circ}$, respectively. For transmit and receive, the quadrature birdcage body coil of the MRI system is used. The magnitude of $\mathrm{H}^{+}$is calculated using the formula $\left|\mathrm{H}^{+}\right|=$ $\left(\cos ^{-1}\left(\left|M_{2}\right| /\left|2 M_{1}\right|\right) / \mu_{0} \gamma T_{\mathrm{RF}}\right)$ where $T_{\mathrm{RF}}$ is the duration of the RF excitation pulse and $\gamma$ is the gyro-magnetic ratio. The MR imaging parameters are TR $=1500 \mathrm{~ms}$, $\mathrm{TE}=5 \mathrm{~ms}$, $\mathrm{FOV}=180 \times 180 \mathrm{~mm}$, raw data matrix size $=128 \times 128$, number of averages $=5$, slice thickness $=5 \mathrm{~mm}$, and number of slices $=8$ (no gap). The experiments are conducted using the 3T (nominal) Siemens Magnetom Trio MR scanner installed in UMRAM (National Magnetic Resonance Research Center) at Bilkent University.

On the other hand, for obtaining the phase of $\mathrm{H}^{+}$, a spin-echo MR image is acquired using the quadrature birdcage body coil of the MRI system. The MR imaging parameters are the same as above except for the nominal flip angle which is chosen to be $90^{\circ}$. The phase of this spin-echo image can be written as

$$
\phi_{s}\left(\mathbf{r}, T_{E}\right)=\phi_{t r}(\mathbf{r})+\int_{0}^{T_{E}} \boldsymbol{\gamma} B_{e} \mathrm{dt}
$$

where $\mathbf{r}$ is the position vector, $T_{E}$ is the echo-time, $\phi_{t r}(\mathbf{r})$ is the transceive phase, and $\int_{0}^{T_{E}} \gamma B_{e} \mathrm{dt}$ is the phase accumulated due to the eddy-currents generated inside the imaging object during the rise-time of the read-out gradient field. $\phi_{t r}(\mathbf{r})$ is the sum of two contributions, namely $\phi_{t r}(\mathbf{r})=\phi_{+}(\mathbf{r})+\phi_{-}(\mathbf{r})$, where $\phi_{+}(\mathbf{r})$ is the phase due to the RF excitation field, $H^{+}$, and $\phi_{-}(\mathbf{r})$ is the phase due to the receive sensitivity distribution. Since spin-echo imaging is used, there is no phase term related to the $B_{0}$ field inhomogenity.

It is known fact that the polarity of $\int_{0}^{T_{E}} \gamma B_{e} \mathrm{dt}$ term depends on the polarity of the read-out gradient, i.e., if $k$-space is scanned

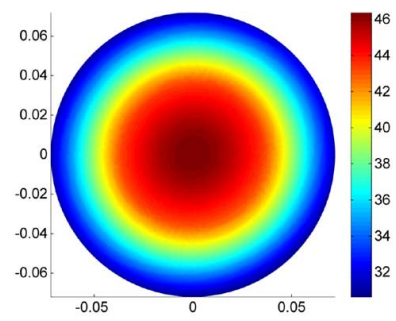

(a)

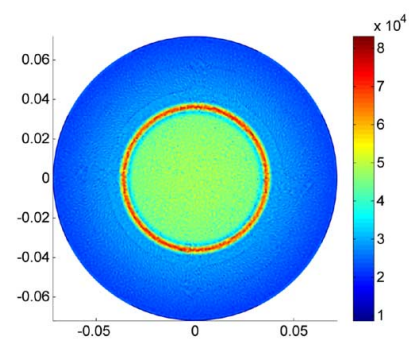

(c)

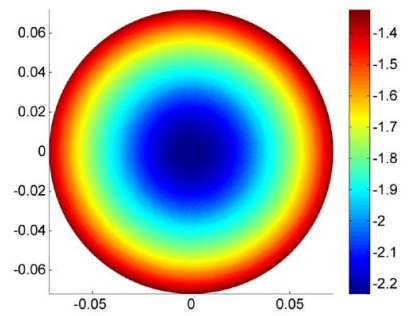

(b)

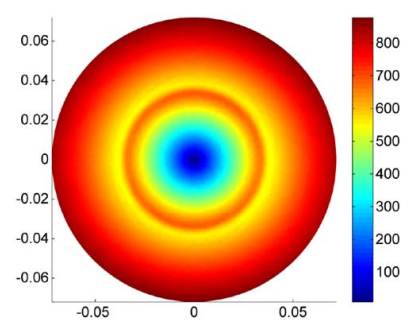

(d)
Fig. 4. Simulation results for the central axial slice of the first simulation phantom: (a) magnitude of $H^{+}(\mathrm{A} / \mathrm{m})$, (b) phase of $H^{+}(\mathrm{rad})$, (c) modulus of $\nabla^{2} H^{+}\left(\mathrm{A} / \mathrm{m}^{3}\right)$, (d) modulus of the convective field $\left(\mathrm{A} / \mathrm{m}^{2}\right)$. Units for axes are meters. Modulus of the convective field has much lower value at the region around the center of the imaging slice, and this region is called as LCF region.

from $k_{x, \max }$ to $-k_{x, \max }$ rather than from $-k_{x, \text { max }}$ to $k_{x, \max }$, this term changes sign (assuming read-out direction is $x$ ). In this study, as suggested in [28], this fact is exploited for obtaining $\phi_{t r}(\mathbf{r})$ as follows: Two phase images are acquired by using spin-echo pulse sequences of different read-out gradient polarities. Their phases are then summed and the resulting phase is halved to obtain $\phi_{t r}(\mathbf{r})$. It is assumed that the transmit and receive phases of a quadrature birdcage coil are very close to each other, i.e., $\phi_{+}(\mathbf{r}) \approx \phi_{-}(\mathbf{r})$ [25], and as a result of this approximation (transceive phase approximation), the phase of $\mathrm{H}^{+}$is calculated as half of the transceive phase, i.e., $\phi_{+}(\mathbf{r}) \approx$ $\phi_{t r}(\mathbf{r}) / 2$.

Complete determination of $\mathrm{H}^{+}$requires the application of two gradient echo sequences and two spin echo sequences which altogether take $4 \times 16=64 \mathrm{~min}$.

3) Obtaining $\mathrm{H}^{+}$on the Nodes of the Triangular Mesh: As discussed above, the MREPT algorithm proposed in this study is triangular mesh based and it is required that $H^{+}$is known on the nodes of the triangular mesh in the imaging slice. In order to obtain $\mathrm{H}^{+}$on the nodes of the triangular mesh, it is necessary that $M_{1}, M_{2}$, and the spin-echo MR images are reconstructed on the nodes as well. In a traditional MRI experiment, MR images are obtained on a rectangular grid by the inverse FFT of the acquired k-space data. Instead, in this study, MR images are directly obtained on the nodes of the triangular mesh from the k-space data by evaluating the inverse discrete Fourier transform at the nodes: The value of a complex MR image at the $k$ th node, $m(k)$ can be expressed as

$m(k)=\frac{1}{N^{2}} \sum_{u=1}^{N} \sum_{v=1}^{N} s(u, v) e^{i 2 \pi \Delta k\left(v x_{k}+u y_{k}\right)}, \quad k=1,2, \ldots, N_{p}$

where $s(u, v)$ denotes the raw data matrix, $\Delta k$ denotes the spatial frequency spacing in $x$ - and $y$-directions $(\Delta k=1 / F O V)$, 

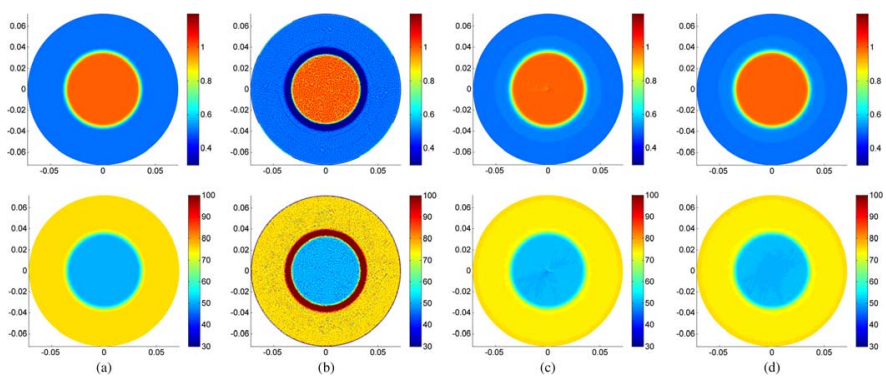

Fig. 5. Reconstruction results for the first simulation phantom: Upper row is for conductivity $\sigma(\mathrm{S} / \mathrm{m})$ and lower row is for relative permittivity $\varepsilon_{\mathrm{r}}$ : (a) true $\sigma$ $\left(\varepsilon_{\mathrm{r}}\right)$, (b) reconstructed $\sigma\left(\varepsilon_{\mathrm{r}}\right)$ using the std-MREPT method, (c) reconstructed $\sigma\left(\varepsilon_{\mathrm{r}}\right)$ using cr-MREPT method, (d) reconstructed $\sigma\left(\varepsilon_{\mathrm{r}}\right)$ using the constrained cr-MREPT method. The spot-like artifact observed in (c) at the center is eliminated when constrained cr-MREPT method is used as shown in (d). Units for axes are meters.

$x_{k}$ and $y_{k}$ denote the $x$ - and $y$ - coordinates of the $k$ th node, $N$ denotes the raw data matrix size, and $N_{p}$ denotes the number of nodes in the imaging slice.

\section{RESULTS}

\section{A. Simulation Results}

1) The Constrained cr-MREPT and the Double-Excitation cr-MREPT Methods: In this section, we present results using simulated data without additive noise. The cross-sectional view of the actual conductivity and permittivity distributions of the first simulation phantom which consist of two concentric cylinders are shown in Fig. 5(a). In the internal boundaries (transition regions), the material properties change in a tapered fashion (not abruptly). The central slice $(\mathrm{z}=0)$ of the phantom is chosen as the slice of interest, and the corresponding $\mathrm{H}^{+}$magnitude, and $\mathrm{H}^{+}$phase distributions are shown in Fig. 4(a) and (b). $\nabla^{2} H^{+}$distribution, and the modulus of the convective field, $|\mathbf{F}|$, are shown in Fig. 4(c) and (d) (note that since $F_{y}=i F_{x}$, $\left.|\mathbf{F}|=\sqrt{\mathbf{F} \cdot \mathbf{F}^{*}}=\sqrt{2}\left|F_{x}\right|=\sqrt{2}\left|F_{y}\right|\right)$. It is observed that the $\nabla^{2} H^{+}$distribution, as expected, has high magnitude in the transition regions. It is also observed that the modulus of the convective field attains very low values around the center and in fact falls to zero at the center. Using these data, $\sigma$ and $\varepsilon$ distributions are reconstructed by applying both the std-MREPT method and also the cr-MREPT method that we have proposed.

The reconstructed $\sigma$ and $\varepsilon$ distributions obtained using the std-MREPT method are shown in Fig. 5(b) and Fig. 6(b) and (d). This method gives good reconstruction results in the regions where $\sigma$ and $\varepsilon$ do not vary but it yields severe errors in the transition regions. This is because std-MREPT method assumes that spatial variations of $\sigma$ and $\varepsilon$ are small in the region of reconstruction and the term involving $\bar{\nabla} u=\bar{\nabla}(1 / \gamma)$ in (8) is not taken into account in this method.

Fig. 5(c) and Fig. 6(a) and (c) shows the results of the cr-MREPT method and the most important advantage of this method seems to be its ability to reliably reconstruct $\sigma$ and $\varepsilon$ distributions everywhere including the transition regions. On the other hand, cr-MREPT method seems to be ill-conditioned (not well-posed) at the origin where a spot-like artifact is observed. This artifact is mainly due to the numerical errors introduced by the region where the modulus of the convective

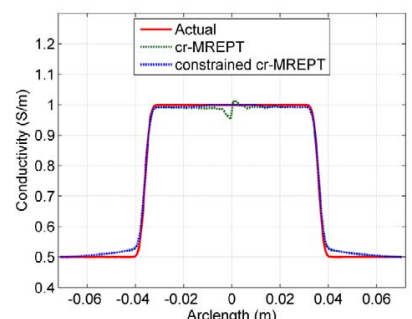

(a)

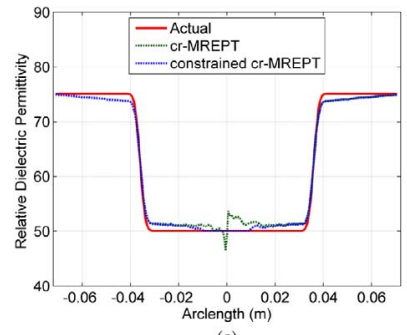

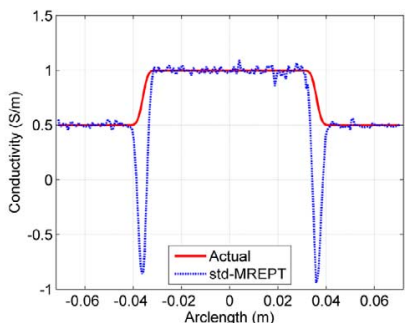

(b)

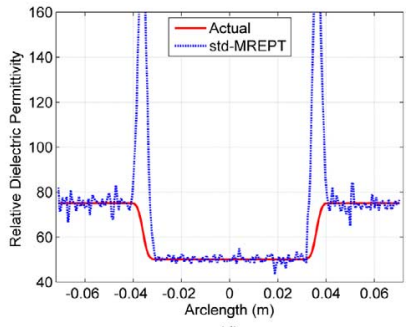

Fig. 6. Line profiles of the reconstructed and the actual conductivity along the $\mathrm{x}$-axis for the first simulation phantom. (a) cr-MREPT and the constrained cr-MREPT are used for the reconstruction. (b) std-MREPT method is used for the reconstruction. (c) and (d) Reconstructed relative permittivity using the same methods as in (a) and (b). Spot-like artifact observed in cr-MREPT reconstructions is eliminated when constrained cr-MREPT is used.

field [in (8)] is nearly zero [shown in Fig. 4(d)]. This region is referred to as the low convection field (LCF) region, and it corresponds to the region where z-component of the total current (conduction current + displacement current) is small.

In order to eliminate the spot-like artifact, we propose two different methods. In the first method, called "constrained cr-MREPT," we first determine the LCF region by observing the modulus of the convective field. In the LCF region, we reconstruct $\sigma$ and $\varepsilon$ using the std-MREPT method [i.e., (9)] which is derived by ignoring the convection term in (8). Then, we apply the cr-MREPT method for the whole domain, whereby $\sigma$ and $\varepsilon$ found by the std-MREPT method in the LCF region are used as a priori knowledge (i.e., as constraint as explained in Section III-A. The resulting reconstructed $\sigma$ and $\varepsilon$ distributions, shown in Fig. 5(d), do not have spot-like artifacts in the LCF region which we have taken to be the circular region of radius $0.01 \mathrm{~m}$ at the center. This improvement in the reconstructions is also observed in the line profiles of reconstructed conductivity and permittivity shown in Fig. 6(a) and (c). However, this method does not give reliable reconstruction results when the LCF region coincides with the boundaries. This is simply because std-MREPT method gives unreliable estimates of the electrical properties in regions where they vary. To cover such cases we propose a second artifact-elimination method as explained below.

In the second method, called "double-excitation cr-MREPT," $\sigma$ and $\varepsilon$ are reconstructed using together two different $\mathrm{H}^{+}$data that have different LCF regions. To explain and test this method the second simulation phantom shown in Fig. 3(b) is used. In this phantom there are three regions, C, D, and E. Regions D and $\mathrm{E}$ are the background regions and region $\mathrm{C}$ represents the anomaly region where $\sigma$ and $\varepsilon$ are different. This anomaly region and its surrounding (union of regions $\mathrm{C}$ and $\mathrm{D}$ ) is our ROI. The actual $\sigma$ and $\varepsilon$ distributions in the ROI are shown in 


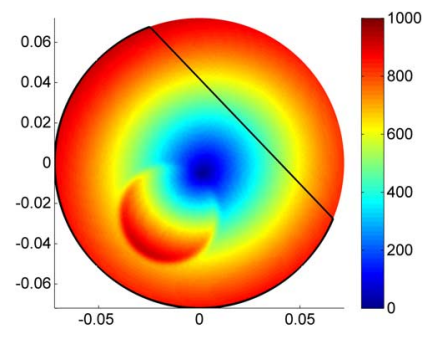

(a)

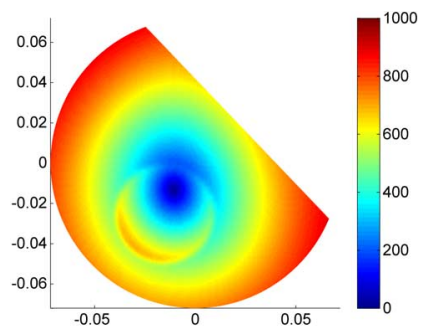

(b)
Fig. 7. Moduli of the convective fields $\left(\mathrm{A} / \mathrm{m}^{2}\right)$ for the second simulation phantom using two different excitations: (a) region $\mathrm{E}$ is included (assigned the same material properties as region $\mathrm{D}$ ), (b) region $\mathrm{E}$ is cut out (assigned material properties of air). ROI (regions C and D) is enclosed by a black border in (a). Convective fields shown in (a) and (b) have different LCF regions in the ROI. Units for axes are meters.

Fig. 8(a). We need to realize two different experiments in which the $\mathrm{H}^{+}$data in the ROI are different. This is achieved by including or excluding region $\mathrm{E}$ in the simulations. In other words in one case region $\mathrm{E}$ is assigned the same material properties as for region $\mathrm{D}$, and in the other case it is assumed to be cut out (or assigned material properties of air). Thus we obtain two different $H^{+}$data for the ROI by modifying the regions external to the ROI. The two different $H^{+}$data, that also have different LCF in the ROI, are shown in Fig. 7(a) and (b). If these $H^{+}$data are used separately for cr-MREPT method, the reconstructions shown in Fig. 9(a) and (b) are obtained and the spot-like artifacts can be observed in the corresponding LCF regions. Using these $H^{+}$data together, (8) is solved via (16) and the resulting reconstructed $\sigma$ and $\varepsilon$ distributions, shown in Fig. 9(c), do not have spot-like artifacts.

Fig. 8(b) shows the reconstruction results for the second excitation of the second simulation phantom using the std-MREPT method. As expected this method has unacceptable performance in the transition regions where the electrical properties are changing. On the other hand, Fig. 8(c) shows the same reconstructions for the second excitation when the constrained cr-MREPT method is used. Note that, as shown in Fig. 7(b), the LCF region for the second excitation is located inside the object where the electrical properties are constant. Therefore, in the LCF region, the std-MREPT method is used to obtain the electrical properties and the constrained cr-MREPT method is then applied in the whole solution domain including the transition regions resulting in successful reconstructions for both $\sigma$ and $\varepsilon$. However, for the first excitation of the second simulation phantom, the LCF region coincides with an internal boundary where the electrical properties are changing as observed from Fig. 7(a) (This is also apparent from Fig. 9(a) where the spot-like artifact occurs at the transition region). In such cases, the std-MREPT method cannot be used in the LCF region and thus the constrained cr-MREPT method would fail to reconstruct the electrical properties in the whole solution domain.

2) Performance of the cr-MREPT Methods Against Noise: The noise tolerance of the cr-MREPT method is investigated by using the second simulation phantom. For this purpose, noise is added to the simulated $H^{+}$data as explained in Section III-B2 for SNR values of 150, 100, and 50. With noisy
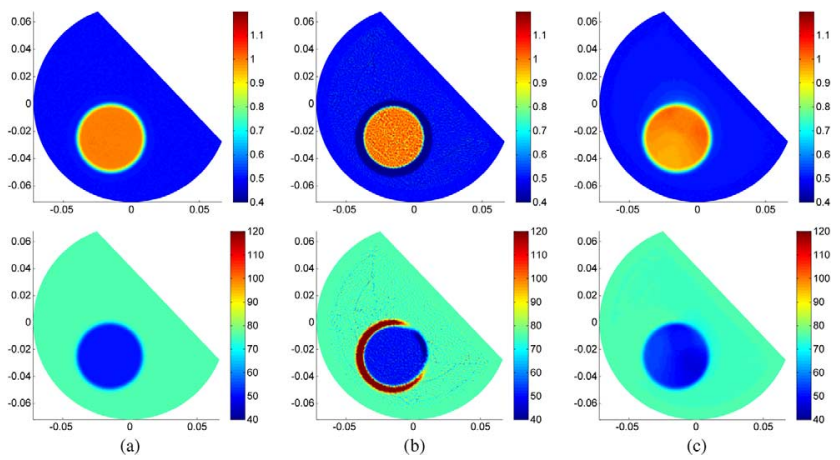

Fig. 8. Reconstruction results for the second simulation phantom: Upper row is for conductivity $\sigma(\mathrm{S} / \mathrm{m})$ and lower row is for relative permittivity $\varepsilon_{\mathrm{r}}$. (a) Actual electrical properties, (b) reconstructions using the std-MREPT method, (c) reconstructions using the constrained cr-MREPT method. Units for axes are meters.
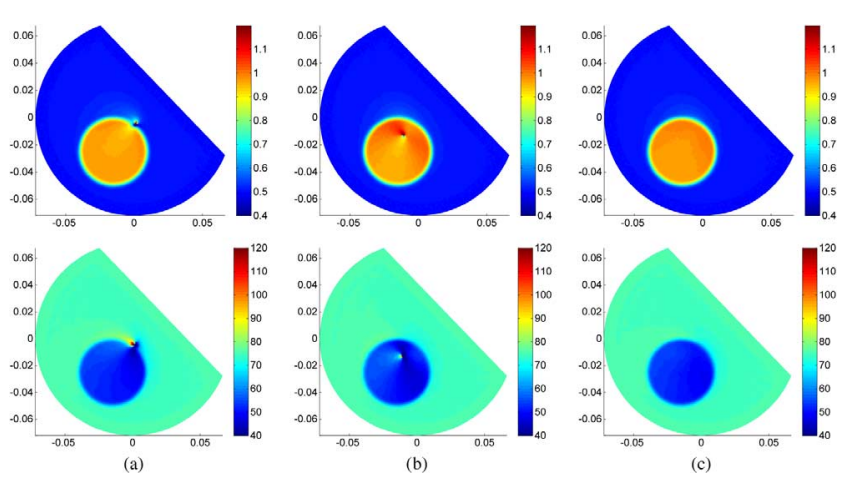

Fig. 9. Reconstruction results for the second simulation phantom: Upper row is for conductivity $\sigma(\mathrm{S} / \mathrm{m})$ and lower row is for relative permittivity $\varepsilon_{\mathrm{r}}$. cr-MREPT method is used (a) for only the first excitation and (b) for only the second excitation. (c) Double-excitation cr-MREPT method is used. The spot-like artifacts observed in (a) and (b) at different locations, are eliminated when double-excitation cr-MREPT method is used as shown in (c). Units for axes are meters.

data even for SNR $=150$ the reconstructed images become totally unacceptable to the extent that the anomaly region is not even distinguished. Therefore, the noise-added $H^{+}$data is low pass filtered before reconstructions are made and the resulting reconstructions are shown in Fig. 10 and Fig. 11. For the noiseless but filtered data, the effect of filtering alone is observed in Fig. 11(a) where double-excitation cr-MREPT method is applied. As expected the transition regions are blunted and widened implying reduction of spatial resolution. (For this case, the relative $L^{2}$ errors for the reconstructed $\sigma$ and $\varepsilon$ are $6.2 \%$ and $3.3 \%$, respectively, as shown in Table I. A significant portion of these errors are due to the low pass filter mentioned above. If the low-pass filter is not used, then for the noiseless case the corresponding relative $L^{2}$ errors are $2.3 \%$ and $1.9 \%$. The filter tapers the variations of $\nabla^{2} H^{+}$in the transition regions and consequently blunts the variations of $\sigma$ and $\varepsilon$ across the internal boundaries and thus relative $L^{2}$ errors increase). As shown in Fig. 10(a) and (b), despite the use of filtering the single-excitation cr-MREPT reconstructions are grossly distorted even for $\mathrm{SNR}=150$. This distortion is mostly profound in and around the LCF regions. Constrained cr-MREPT method, on the other hand, gives acceptable reconstruction images [Fig. 10(c)] for SNR $=150$ when the second 

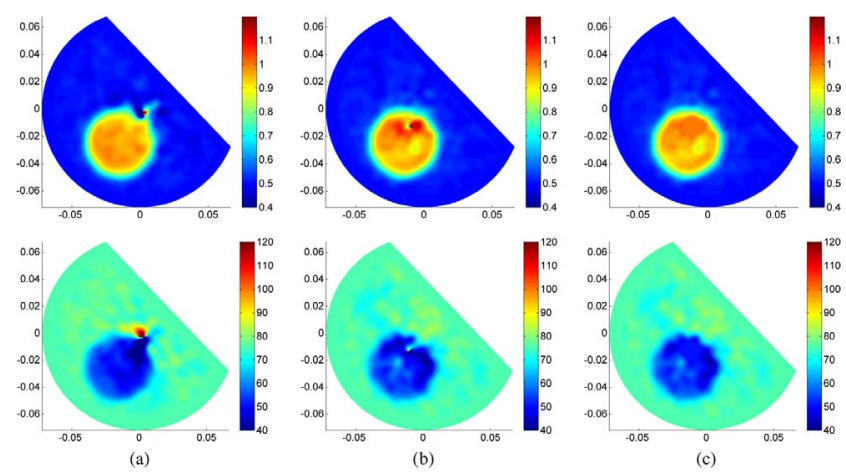

Fig. 10. Reconstruction results of cr-MREPT when noise corresponding to SNR of 150 is added: cr-MREPT method is used (a) for only the first excitation and (b) for only the second excitation. (c) Constrained cr-MREPT method is used. Upper row is for conductivity $\sigma(\mathrm{S} / \mathrm{m})$ and lower row is for relative permittivity $\varepsilon_{\mathrm{r}}$. Units for axes are meters.
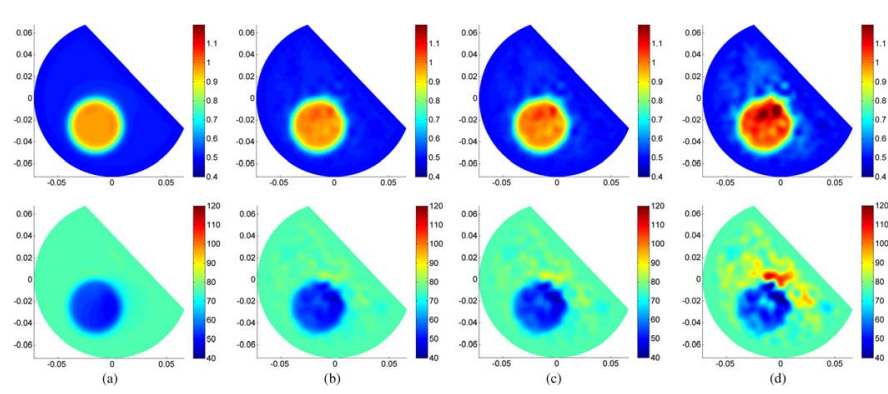

Fig. 11. Double excitation cr-MREPT reconstruction results for the second simulation phantom when noise corresponding to SNRs of $\infty, 150,100$, or 50 is added to each $H^{+}$data obtained for the two excitations. Upper row is for conductivity $\sigma(\mathrm{S} / \mathrm{m})$ and lower row is for relative permittivity $\varepsilon_{\mathbf{r}}$. Units for axes are meters.

excitation data is used. Fig. 11 depicts the performance of the double-excitation cr-MREPT method for SNR $=150,100$, and 50 . The relative $L^{2}$ errors in the reconstructed images using both the constrained and double-excitation cr-MREPT method for different SNR values are listed in Table I. In general, we have found that using the double-excitation cr-MREPT method, the artifacts appearing around the LCF region are significantly reduced except for very low SNR. Despite the use of double-excitation, as SNR is reduced, some systematic changes occur in and around the LCF regions. We think this is due to enlargement of the LCF regions as a result of interaction with noise. The constrained cr-MREPT is also successful but, as SNR is decreased, LCF region may spread into the transition regions making the application of constrained cr-MREPT unfeasible.

3) Effects of the Transceive Phase Approximation on cr-MREPT Reconstructions: The phase of the RF excitation field $H^{+}$, i.e., $\phi_{+}(\mathbf{r})$, is calculated exactly, within the limits of the numerical methods, and is used in MREPT algorithms when electrical property reconstructions are made from simulated data. However, in real experiments we cannot measure $\phi_{+}(\mathbf{r})$ directly and we approximate it as half of the transceive phase, $\phi_{t r}(\mathbf{r})$. It is necessary to assess the effect of this TPA on the reconstructions. The cr-MREPT reconstructions obtained when the transmit phase is approximated as half of the transceive phase are shown in Fig. 12 for the second simulation phantom.
TABLE I

$L^{2}$ ERRORS IN $\sigma$ AND $\varepsilon$ RECONSTRUCTED USING THE CR-MREPT METHODS WHEN NOISE CORRESPONDING TO DIFFERENT SNR VALUES ARE ADDED TO $H^{+}$(LPF: LOW PASS FILTER)

\begin{tabular}{|c|c|c|c|c|c|}
\cline { 3 - 6 } \multicolumn{2}{c|}{} & \multicolumn{4}{c|}{ Relative $L^{2}$ error $(\%)$} \\
\cline { 3 - 6 } \multicolumn{2}{c|}{} & $\begin{array}{c}\text { Double-excitation } \\
\text { cr-MREPT }\end{array}$ & \multicolumn{2}{c|}{$\begin{array}{c}\text { Constrained } \\
\text { cr-MREPT }\end{array}$} \\
\hline SNR & LPF & $\mathrm{E}_{L^{2}}(\sigma)$ & $\mathrm{E}_{L^{2}}(\varepsilon)$ & $\mathrm{E}_{L^{2}}(\sigma)$ & $\mathrm{E}_{L^{2}}(\varepsilon)$ \\
\hline$\infty$ & No & 2.3 & 1.9 & 2.4 & 2.0 \\
\hline$\infty$ & Yes & 6.2 & 3.3 & 6.4 & 3.3 \\
\hline 150 & Yes & 6.4 & 4.0 & 7.2 & 4.4 \\
\hline 100 & Yes & 6.8 & 5.2 & 8.0 & 5.9 \\
\hline 50 & Yes & 10.9 & 11.7 & 10.6 & 10.8 \\
\hline
\end{tabular}
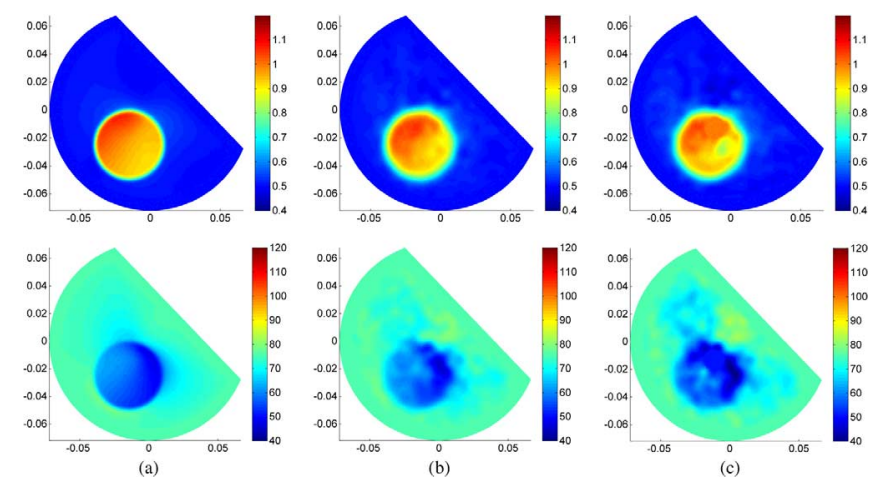

Fig. 12. Effect of TPA on cr-MREPT reconstructions. (a) Noise-free case with double-excitation cr-MREPT method, (b) SNR $=150$ case with double excitation cr-MREPT method, and (c) SNR $=150$ case with constrained cr-MREPT method. Units for axes are meters.

For the noiseless case, using the double-excitation cr-MREPT method, reconstructions are shown in Fig. 12(a). It is found that $\sigma$ and $\varepsilon_{r}$ values in the inner object vary between $0.92-1.08$ $\mathrm{S} / \mathrm{m}$ and $46-63$, respectively. If TPA is not applied, as measured from the reconstructions shown in Fig. 9(c), the $\sigma$ and $\varepsilon_{r}$ values in the inner object vary between $0.98-1.02 \mathrm{~S} / \mathrm{m}$ and 54-48, respectively. The increased variation observed with the introduction of TPA is in the form of a low frequency trend. However the success of cr-MREPT in reconstructing transition regions is not lost when TPA is used. For the SNR $=150$ case, reconstructions of both the double-excitation and the constrained cr-MREPT methods, as shown in Fig. 12(b) and (c), are effected by TPA again by the introduction of low frequency trends. The relative $L^{2}$ errors in the reconstructions with the TPA are given in Table II, and when compared with the results of Table I (which has the results without TPA) it is observed that the relative $L^{2}$ errors somewhat increase, but are still in the same range especially when noisy data is used.

4) Application of the cr-MREPT Method to a Head Model: In order to observe the performance of the cr-MREPT method under more realistic conditions we have applied it to a head model. Fig. 13 has a 2-D axial brain slice segmented into five tissues, namely, cerebro spinal fluid (CSF), white matter (WM), gray matter (GM), skull, and scalp. Table III has the electrical properties assigned to these tissues. Also in Fig. 13 a padding region is shown which is assumed to be padded to the head in order to obtain measurements of a second excitation. This padding is assigned a conductivity value close to that of gray matter, and a 
TABLE II

$L^{2}$ ERRORS IN $\sigma$ AND $\varepsilon$ RECONSTRUCTED BY THE CR-MREPT METHODS WHEN NOISE CORRESPONDING TO DIFFERENT SNR VALUES ARE ADDED TO $\mathrm{H}^{+}$AND WHEN TPA IS USED. (LPF: LOW PASS FILTER)

\begin{tabular}{|c|c|c|c|c|c|}
\cline { 3 - 6 } \multicolumn{2}{c|}{} & \multicolumn{4}{c|}{ Relative $L^{2}$ error $(\%)$} \\
\cline { 3 - 6 } \multicolumn{2}{c|}{} & $\begin{array}{c}\text { Double-excitation } \\
\text { cr-MREPT }\end{array}$ & \multicolumn{2}{c|}{$\begin{array}{c}\text { Constrained } \\
\text { cr-MREPT }\end{array}$} \\
\hline SNR & LPF & $\mathrm{E}_{L^{2}}(\sigma)$ & $\mathrm{E}_{L^{2}}(\varepsilon)$ & $\mathrm{E}_{L^{2}}(\sigma)$ & $\mathrm{E}_{L^{2}}(\varepsilon)$ \\
\hline$\infty$ & No & 4.3 & 6.5 & 4.3 & 5.9 \\
\hline$\infty$ & Yes & 7.3 & 6.9 & 7.1 & 6.0 \\
\hline 150 & Yes & 7.4 & 7.0 & 8.0 & 7.5 \\
\hline 100 & Yes & 7.7 & 7.4 & 8.7 & 8.8 \\
\hline 50 & Yes & 11.3 & 11.8 & 11.2 & 13.1 \\
\hline
\end{tabular}

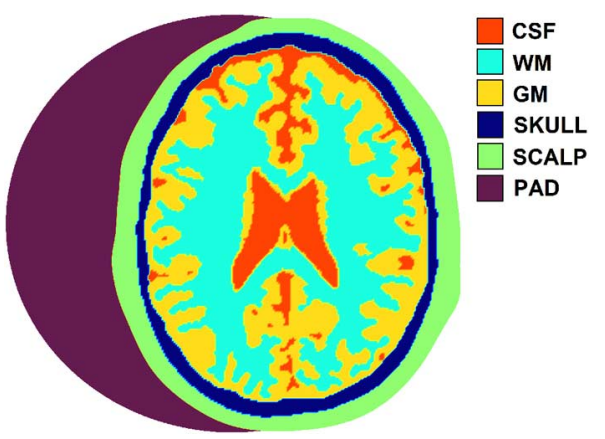

Fig. 13. Illustration of the simplified head model and the pad which is attached to one side of the head.

TABLE III

Electrical Properties of the Head Model AND the PaD

\begin{tabular}{c|cc}
\hline Tissue & $\sigma(S / m)$ & $\varepsilon_{r}$ \\
\hline CSF & 2.14 & 84 \\
WM & 0.34 & 52 \\
GM & 0.59 & 73 \\
Skull & 0.12 & 21 \\
Scalp & 0.54 & 62 \\
Pad & 0.6 & 100 \\
\hline
\end{tabular}

permittivity value of slightly higher than CSF. Simulations are performed using the same birdcage coil given in Fig. 2. The 2-D head slice with padding shown in Fig. 13 is extruded in z-direction, by $17.5 \mathrm{~cm}$, to form a 3-D geometry and the final 3-D object is placed in the birdcage coil. Fig. 14(b)-(e) depicts the results of cr-MREPT for the first excitation (i.e., without padding), cr-MREPT for the second excitation (i.e., with padding), double excitation cr-MREPT, and constrained cr-MREPT for the first excitation, respectively. As seen in Fig. 14(b) and (c) the presence of padding significantly shifts the LCF region as apparent from the shift of the spot-like artifact observed for single excitation cr-MREPT reconstructions. Both the double-excitation and constrained cr-MREPT methods give very satisfactory results especially for the conductivity. The permittivity reconstructions have more error especially in the CSF regions which have higher $\sigma$ and $\varepsilon_{r}$.

In the reconstruction results given in Fig. 14, the transmit phase is assumed to be known. The effect of TPA on the cr-MREPT reconstructions is also investigated for the head model. Fig. 15(a) and (b) shows the actual transmit phase $\left(\phi_{+}(\mathbf{r})\right)$ and the error introduced by TPA for the excitations without and with padding, respectively. Without padding the error in the transmit phase introduced by TPA is about $\pm 3^{\circ}$ and it increases to $\pm 5^{\circ}$ increased when padding is used. Fig. 15(c) and (d) shows the reconstructions obtained when TPA is applied when using double-excitation cr-MREPT, and constrained cr-MREPT for the first excitation, respectively. The relative $L^{2}$ errors in the reconstructions with and without the transceive phase approximation are given in Table IV. With the TPA, the relative $L^{2}$ error is slightly increased for the conductivity and there is no observable difference comparing the reconstructed conductivities in Fig. 14(d) and (e) with the ones in Fig. 15(c) and (d). However, the impact of TPA on the permittivity reconstructions seems to be higher as evident from the relative $L^{2}$ error values in Table IV. Especially in the CSF region, where the $\sigma$ and $\varepsilon_{r}$ are higher, the error is introduced in permittivity by TPA is more apparent as shown in Fig. 15(c) and (d). Fig. 15(e) shows the reconstruction results when noise is added to the simulated $H^{+}$data of the head model in an amount corresponding to an MR SNR of 100, and TPA is also applied. As before, when noise is added, a low pass filter with Gaussian kernel with standard deviation $0.0032 \mathrm{~m}$ is applied before reconstructions are undertaken. It is observed that the noise is very well tolerated by the cr-MREPT algorithm and however due to filtering transition regions are widened.

\section{B. Experimental Results}

Experiments are first conducted using the first experimental phantom. The same low pass filter, which is used for noisy simulated data as mentioned above, is applied also to the measured $H^{+}$. Fig. 16(a) and (b) shows the measured and filtered $H^{+}$ magnitude and phase $\left(\phi_{+}(\mathbf{r})\right)$. Fig. 16(c) and (d) shows the modulus of $\nabla^{2} H^{+}$, and the modulus of the convective field obtained from the filtered $H^{+}$. In obtaining $\phi_{+}(\mathbf{r}) \approx \phi_{t r}(\mathbf{r}) / 2$ we have also eliminated the phase accumulated due to the eddy-currents as explained in Section III-C2. For comparison purposes, half of the phase accumulated due to the eddy currents, i.e., $(1 / 2) \int_{0}^{T_{E}} \gamma B_{e} \mathrm{dt}$, and half of the phase of the spin-echo image, $\phi_{s}\left(\mathbf{r}, T_{E}\right) / 2$, are shown in Fig. 16(e) and (f), respectively. It is observed that the phase accumulated due to the eddy currents is significant (peak to peak level of $0.4 \mathrm{rad}$ ) compared to $\phi_{+}(\mathbf{r})$ (peak-to-peak level of $0.7 \mathrm{rad}$ ). These results justify the need for the elimination of eddy current induced phase when finding $\phi_{+}(\mathbf{r})$.

Reconstructed conductivity distributions are first obtained by applying std-MREPT and Voigt's methods which are explained in the introduction, and are shown in Fig. 17(a) and (b), respectively. Similar to the simulation case, the result of std-MREPT method has severe errors on the internal boundaries. Since stdMREPT method is a point-wise method and it depends on the second derivatives of $H^{+}$, this method is very sensitive to noise and the reconstructed conductivity distribution is very noisy, as shown in Fig. 17(a). However, since Voigt's method has an integral based formula and it depends on the first derivatives of $\mathrm{H}^{+}$only, this method gives a less noisy reconstruction result as shown in Fig. 17(b). Voigt's method still has severe errors in the transition regions. 


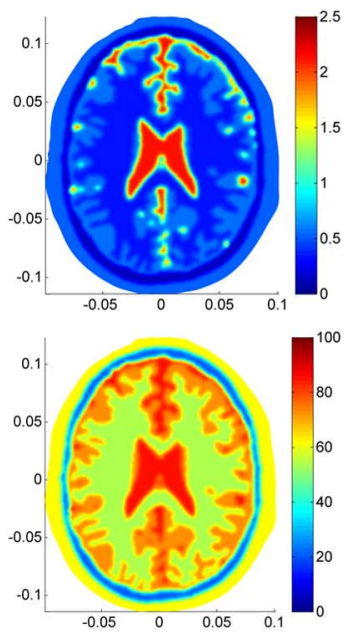

(a)
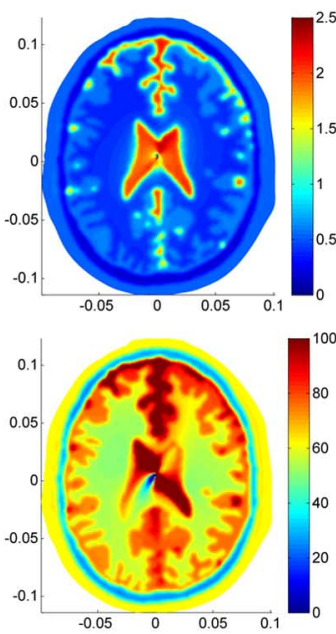

(b)
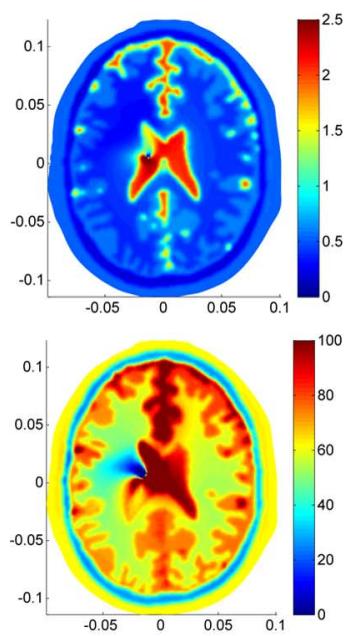

(c)
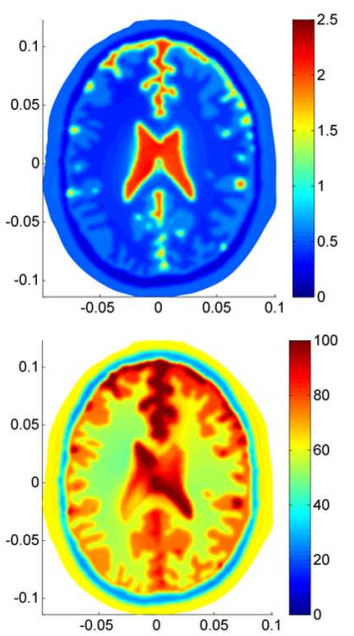

(d)

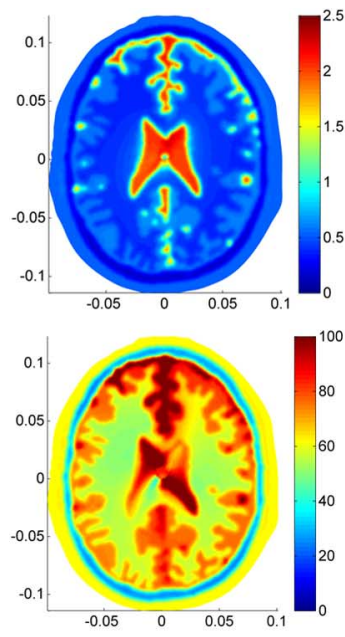

(e)

Fig. 14. Reconstruction results for the head model (Upper row is for conductivity $\sigma(\mathrm{S} / \mathrm{m})$ and lower row is for relative permittivity $\varepsilon_{\mathrm{r}}$ ). (a) Actual electrical properties. (b) cr-MREPT reconstructions using the first excitation. (c) cr-MREPT reconstructions using the second excitation. (d) Double-excitation cr-MREPT method results. (e) Constrained cr-MREPT method results. Units for axes are meters.
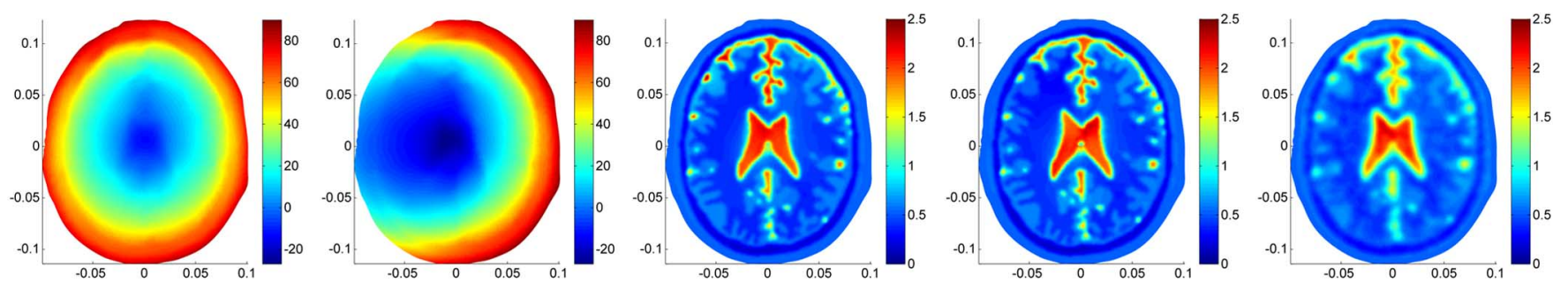

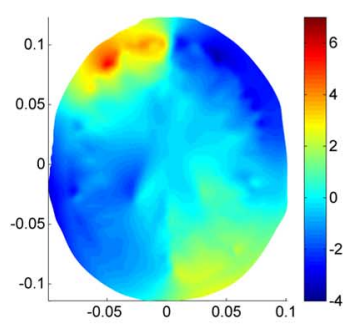

(a)

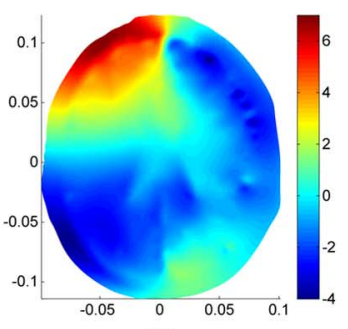

(b)

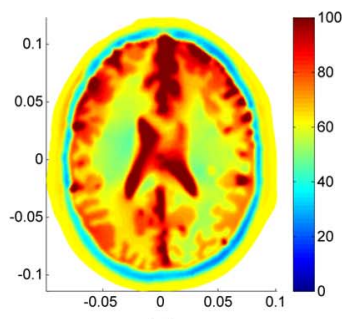

(c)

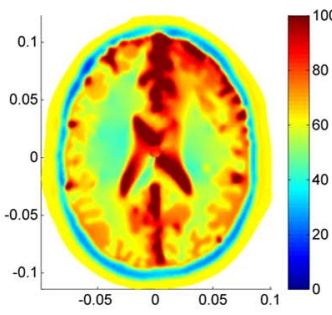

(d)

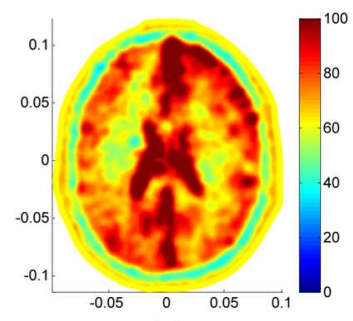

(e)

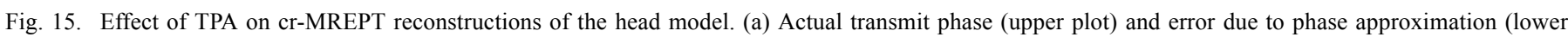

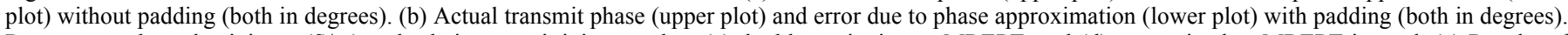

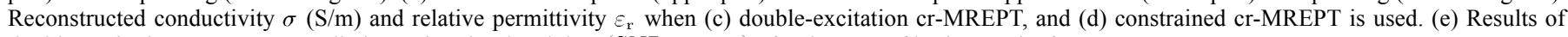
double-excitation cr-MREPT applied to noisy simulated data $(\mathrm{SNR}=100)$ after low pass filtering. Units for axes are meters.

TABLE IV

EFFECT OF TPA FOR THE BRAIN MODEL $L^{2}$ ERRORS IN $\sigma$ AND $\varepsilon$

\begin{tabular}{|c|c|c|c|c|}
\cline { 2 - 5 } \multicolumn{1}{c|}{} & \multicolumn{4}{c|}{ Relative $L^{2}$ error (\%) } \\
\cline { 2 - 5 } & $\begin{array}{c}\text { Double-excitation } \\
\text { cr-MREPT }\end{array}$ & \multicolumn{2}{c|}{$\begin{array}{c}\text { Constrained } \\
\text { cr-MREPT }\end{array}$} \\
\cline { 2 - 5 } & $\mathrm{E}_{L^{2}}(\sigma)$ & $\mathrm{E}_{L^{2}}(\varepsilon)$ & $\mathrm{E}_{L^{2}}(\sigma)$ & $\mathrm{E}_{L^{2}}(\varepsilon)$ \\
\hline Actual $H^{+}$ & 8.3 & 11.8 & 9.1 & 13.5 \\
\hline$H^{+}$with TPA & 11.3 & 19.2 & 10.7 & 18.6 \\
\hline
\end{tabular}

On the other hand, cr-MREPT is very successful in reconstructing the boundary transitions as shown in Fig. 17(c). However, the result of cr-MREPT method has a spot-like artifact in the LCF region. When the constrained cr-MREPT method, which is explained in Section IV-A, is applied, the reconstructed conductivity shown in Fig. 17(d) is obtained and it does not have a spot-like artifact. In this method, a circular region with radius of $0.007 \mathrm{~m}$ which encloses the spot-like artifact region is first selected. In this region, the averages of the electrical properties found using std-MREPT method are calculated. These average values are used for this region as a constraint (a priori knowledge) in the constrained cr-MREPT method. The average conductivity values for the reconstructed distribution shown in Fig. $17(\mathrm{~d})$ are $0.93 \mathrm{~S} / \mathrm{m}$ for the inner object and $0.43 \mathrm{~S} / \mathrm{m}$ for the background. These values are consistent with the estimated values given in the section on phantom preparation. When applying the cr-MREPT method, the average conductivity and permittivity values obtained by the std-MREPT method at the outer boundary are used for assigning the Dirichlet boundary condition required in solving (8).

Experiments are then performed using the second experimental phantom. Fig. 18(a) and (b) shows the moduli of the 

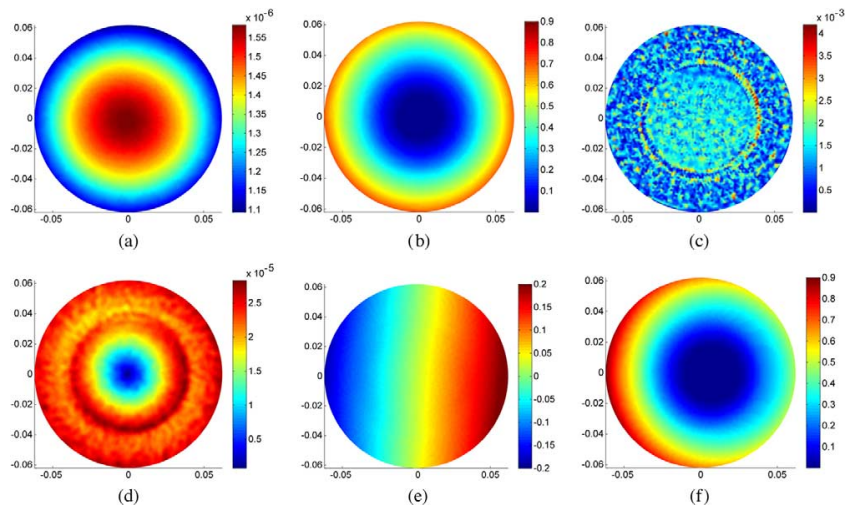

Fig. 16. For the axial slice of the first experimental phantom, (a) magnitude of $B_{1}^{+}$(Tesla), (b) phase of $B_{1}^{+}$(rads), (c) modulus of $\nabla^{2} B_{1}^{+}\left(\mathrm{T} / \mathrm{m}^{2}\right)$, (d) modulus of the convective field $(\mathrm{T} / \mathrm{m}),(\mathrm{e})$ half of the phase accumulated due to the eddy currents, (f) half of the transmit phase if the eddy-current phase is not eliminated. Units for axes are meters.

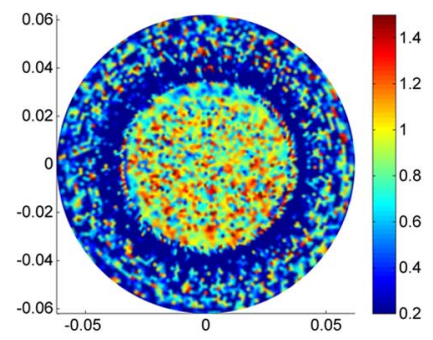

(a)

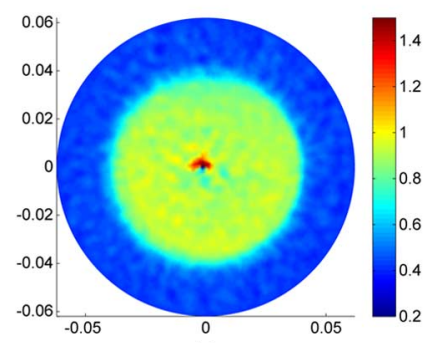

(c)

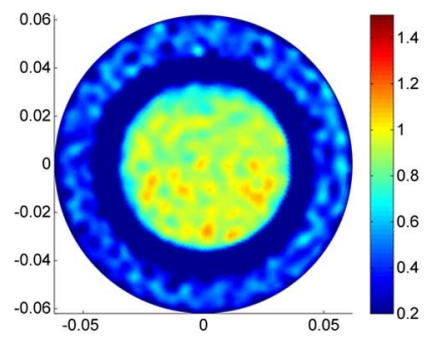

(b)

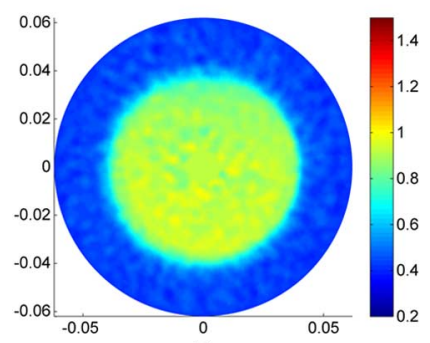

(d)
Fig. 17. Reconstructed conductivity distributions for the first experimental phantom using (a) std-MREPT method, (b) Voigt's method, (c) cr-MREPT method, (d) constrained cr-MREPT method. Units for axes are meters.

convective fields for the first and second excitations which are obtained with and without segment E, respectively. As shown in Fig. 18, these convective fields have different LCF regions that do not coincide with each other. Using the calculated $\nabla^{2} H^{+}$ data of the two cases together, double excitation cr-MREPT method is applied and the reconstructed conductivity distribution is obtained, as shown in Fig. 18(c). Outer boundary conditions are again taken from the results of the std-MREPT method. As expected, the reconstructed conductivity distribution does not have spot-like artifacts, and the boundary transitions are well constructed. The average reconstructed conductivity values are $0.99 \mathrm{~S} / \mathrm{m}$ for the inner object and 0.45 $\mathrm{S} / \mathrm{m}$ for the background, again similar to the estimated values.

We have also conducted experiments with the third experimental phantom which has relatively more complex geometry, as shown in Fig. 3(c). For the conductivity reconstruction, the double-excitation cr-MREPT method is applied. For comparison, the same phantom geometry is also modeled in the simulation environment and the conductivity is reconstructed. Top row of Fig. 19(a) and (b) shows the modulus of the convective field calculated using the experimental data for the first and second excitation, respectively, whereas in the bottom row the convective fields calculated in the simulations are shown. A low pass filter with Gaussian kernel with standard deviation 0.0032 $\mathrm{m}$ is applied when processing both the simulated and the experimental data. It is observed that the convective field distributions in the experiments and in the simulations have similar trends, and in the second excitation the location of the LCF region is similarly shifted. Fig. 19(c) shows the conductivity reconstructions obtained using the experimental data (top row) and using the simulated data (bottom row). The average reconstructed conductivity values are $1.22 \mathrm{~S} / \mathrm{m}$ for the inner objects and $0.62 \mathrm{~S} / \mathrm{m}$ for the background, again similar to the estimated values.

\section{DISCUSSION}

Previously developed practical MREPT algorithms reconstruct electrical properties in regions where $\sigma$ and $\varepsilon$ values vary slowly [25], [26], [28]. In this study, we have proposed a novel algorithm named convection-reaction equation based MREPT (cr-MREPT) which reconstructs $\sigma$ and $\varepsilon$ also in transition regions such as boundaries of internal objects. However, spot-like artifacts are observed in the LCF regions where the convection field is low. To eliminate these artifacts, we have proposed two different methods named as "constrained cr-MREPT" and "double-excitation cr-MREPT." We have validated these MREPT methods using both simulated and experimental data.

The "constrained cr-MREPT" method has the advantage that it can be applied even for single-excitation data but it has the limitation that it cannot be applied in the LCF regions which have varying $\sigma$ and $\varepsilon$. The "double-excitation cr-MREPT" method, however, can be applied to such cases as well. In double-excitation cr-MREPT two different $\mathrm{H}^{+}$ measurements are obtained such that the LCF regions are different. In this study, we have proposed "padding" to obtain two different excitations, one obtained without padding and the other obtained with padding. In our experimental studies, we have realized "padding" by cutting part of the phantoms and then repeating the experiments. In our head simulation phantom, we have envisaged that padding material is attached to one side of the head. We have obtained successful reconstructions with the head phantom using padding material, the electrical properties of which are not very different from the electrical properties of head tissues. Studies which have used passive materials to improve aspects of MRI by effecting the distribution of radio-frequency electromagnetic fields, have led to the development of padding materials in the form of gels and slurries [55]-[57]. Although many of such padding materials are of low conductivity and high permittivity, high conductivity gels $(17 \mathrm{~g} / \mathrm{L} \mathrm{NaCl})$ have also been proposed and used [57]. Conductive padding, in general, compromises SNR [57]. However for the purpose of altering the position of the LCF region, our preliminary studies indicate that conductive padding may be more instrumental. Another effect of padding 

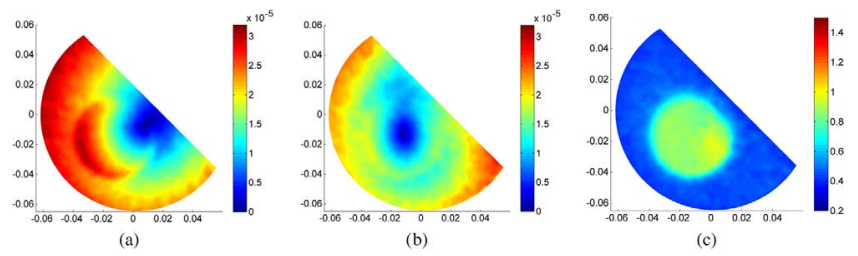

Fig. 18. For the central axial slice of the second experimental phantom, (a) modulus of the convective field $(\mathrm{T} / \mathrm{m})$ for the first excitation, (b) modulus of the convective field $(\mathrm{T} / \mathrm{m})$ for the second excitation, (c) reconstructed conductivity $(\mathrm{S} / \mathrm{m})$ distribution using double-excitation cr-MREPT method. Convective fields shown in (a) and (b) have different LCF regions. Units for axes are meters.
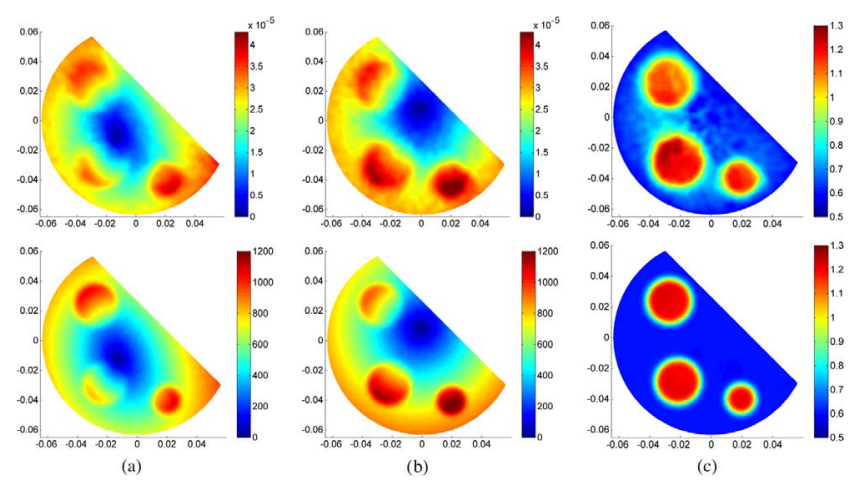

Fig. 19. For the central axial slice of the third phantom, experimental (upper row) and simulated (lower row) (a) modulus of convective field for the first excitation, (b) modulus of convective field for the second excitation, (c) reconstructed conductivity $(\mathrm{S} / \mathrm{m})$ distributions when double-excitation cr-MREPT method is used. Units for axes are meters. Top row convective field units are $\mathrm{T} / \mathrm{m}$ and bottom row convective field units are $\mathrm{A} / \mathrm{m}^{2}$.

which must be taken into consideration is whether the TPA is still applicable when padding is used. The results that we have presented in Section IV-A4 show that, at least for the head phantom and the padding material that we have used in this section, the TPA is still applicable. Although the determination of the exact shape, position, material, and electrical properties of a contacting object to be used for padding is the subject of further studies, the results we have demonstrated in this paper show that double-excitation cr-MREPT using padding is a feasible technique.

In order to realize a second excitation for the double-excitation cr-MREPT method, instead of padding an additional transmit channel (coil) may be used so that the LCF region is shifted. To set up a second matrix equation, as in (15) and (16), we need to obtain the complex $H^{+}$map for the additional coil. If this coil is used for both transmit and receive, then the transceive phase approximation may not hold. As a matter of fact, to the knowledge of the authors, a birdcage coil driven in quadrature mode (or some of its variants like the TEM coil), is the only coil configuration for which the TPA is applicable. As a solution to this problem, for the second excitation one may transmit from the additional coil and receive from the birdcage coil, since the receive phase of the birdcage coil is already known from the first excitation data. It must be noted however that in designing the additional coil one must also be careful about minimizing $H_{z}$ in the slice of interest. The design and application of additional coils for double excitation cr-MREPT will be the focus of future studies.
In this study, the derived convection-reaction equation of MREPT is solved using a triangular mesh based finite difference method. We have used the mesh generation facility of COMSOL Multiphysics in order to obtain a triangular mesh. The solution of the equation itself can also be done by FEM or other numerical methods. In our previous studies, we developed a convection equation-based formulation for MREIT and its numerical solution was based on FEM [58]. Some specific problems which arise when a commercial FEM package is used for the solution are discussed in [58]. We have also applied a FEM-based solution method for constrained cr-MREPT [59]. The extension of FEM to the double excitation cr-MREPT and the use of regularization and stabilization methods will be considered in future studies.

Mesh size is an important factor from the point of view of artifacts and errors. We have found that the spot-like artifact which appears in LCF regions, when especially single-excitation cr-MREPT is used, can be significantly eliminated in the simulations if a very fine mesh is used. One reason for this is that using a fine mesh the solution of the fields become more accurate but another reason is that the first and second derivatives of $\mathrm{H}^{+}$are also calculated more accurately. We have observed this behavior especially when we used some 2-D simulations where we can make the mesh extremely fine (element size of $0.2 \mathrm{~mm}$ ). In such cases the spot like artifact disappears almost completely even in single-excitation cr-MREPT. Also, when high resolution 2-D simulations were made using the head phantom the errors that we have observed especially for the permittivity reconstructions shown in Figs. 14 and 15 disappeared. However, using an extremely fine mesh may not be feasible nor useful in practice because of several reasons. 1) When simulations involving the solution of the forward problem for the 3-D loaded birdcage coil are considered, excessive memory requirements arise as explained below. 2) Although an extremely fine mesh eliminates the spot-like artifacts for the noise-free cases, these artifacts reappear when noise is added to the data. 3) For experimental data the resolution is determined by the resolution of the MR system and therefore in the calculation of the first and second derivatives of $\mathrm{H}^{+}$we are limited by the MR resolution anyway. In our forward problem simulations, we have used a mesh element size of $1 \mathrm{~mm}$ in the slices of interest for the first, second, and third simulation phantoms whereas for the head model $1.4 \mathrm{~mm}$ is used. Mesh element size is increased gradually for more distant slices in order to meet the memory requirements. For example, for the first three simulation phantoms the number of degrees-of-freedom was 11,048,442 and 55 GB RAM was necessary. We have used HP Z800 workstation with Intel Zeon X5675 $3.07 \mathrm{GHz}$ dual processors (12 cores) and with 64 GB RAM. Thus it was not possible to use finer meshes. Solution of one forward problem took $43 \mathrm{~min}$. In solving the inverse problem, we did not have any memory problem since reconstructions were made only for the slice of interest and 41619 elements were sufficient. One complete admittivity reconstruction, including the low-pass filtering of $\mathrm{H}^{+}$, calculation of the derivatives of $H^{+}$, and solving (16) took $225 \mathrm{~s}$.

Noise tolerance of our algorithm is also investigated for different noise levels. Since the Laplacian operation, used in finding $\nabla^{2} H^{+}$, amplifies the noise, the cr-MREPT method is 
relatively sensitive to noise. A low pass filter with Gaussian kernel with standard deviation $0.0032 \mathrm{~m}$ is applied when processing both the noisy simulated data and the experimental data. This filtering causes the transition regions, where electrical properties vary, to appear wider in the reconstructed images. Therefore, in determining the standard deviation of the Gaussian kernel, this tradeoff between having less noisy reconstructions and having higher spatial resolution must be taken into consideration.

In this study, we used the TPA to estimate the phase of the $\mathrm{H}^{+}$field, since we used a quadrature birdcage coil. In fact, the limits of the TPA have been studied for the birdcage coil and it has been argued that the $H^{+}$phase is exactly equal to half the transceive phase in some situations, e.g., circular symmetry in electrical properties [25], [26], [28], [29]. Recently, several groups have investigated the mapping of the transmit phase for multi-channel transmit/receive coil arrays. In these studies, the transmit or receive phase distribution of a certain coil is taken as reference and all the other phases are determined with respect to this [30], [31], [34]. These studies also involve the reconstruction of electrical properties, but their algorithms are confined to regions where the electrical properties vary slowly. Another recent study using a multi-channel array, has addressed the problem of reconstruction also in transition regions, but it must still be assessed under real experimental conditions [35]. The cr-MREPT method that we have developed successfully handles the reconstruction in the transition regions and it is applicable to the widely-used birdcage coil. Furthermore, since multiple $H^{+}$distributions can be obtained using a multi-channel array, and since their LCF regions may be different, the cr-MREPT method can be applied, to advantage, using all these $H^{+}$distributions simultaneously. Further research is needed on this topic.

In the cr-MREPT algorithm, the z-component of the magnetic field intensity $H_{z}$ is neglected. In the case of a RF birdcage coil, $H_{z}$ is generated mainly by the end-rings of the RF birdcage coil. In the central slice, the magnitude of $H_{z}$ is small in the following two cases. 1) $H_{z}$ generated by the end-rings of the opposite sides mostly cancel each other at the central slice especially for a symmetric situation in the $\mathrm{z}$-direction with respect to the central slice. 2) The end-rings are far away from the central slice. In our simulation and experimental phantoms, there is z-symmetry and also the end-rings are distant from the central slice since we have used a body coil. Errors in MREPT measurements gradually increase as slices move off-center, especially if short birdcage coils are used. This limits the applicability of the technique for multi-slice MREPT especially using short head coils which are preferable for SNR purposes. It has been argued by Zhang et al. that when TEM coils, which do not have end-ring currents, are used, better reconstruction results with higher accuracy can be obtained [27]. Another suggestion for minimizing $H_{z}$ effects is made by Katscher et al., who propose to estimate $H_{z}$ from a full model including the birdcage coil and the patient, and/or to find it by iterative computation [32].

In our simulation and experimental studies, we have used phantoms which are geometrically (electrically) invariant in the $\mathrm{z}$-direction. Variations in z-direction, such as in in vivo applications might be expected to influence the accuracy of results.
In this study we have not addressed this issue. If z-variation does not occur exactly on the imaging slice then as can be seen from (7), $\partial u / \partial z$ drops and the slice imaging problem reduces to 2-D. In other words, z-variations distant from the slice of interest do not affect the cr-MREPT reconstructions for that slice. If z-variation is exactly on the slice of interest, then several approaches might be suggested.1) Equation (7) can be handled as a 3-D problem provided that multi-slice data is acquired and the boundary conditions for the 3-D ROI are properly assigned. 2) Data from two excitations can be used such that two different sets of coefficients for (7) are obtained. By an appropriate linear combination of the two equations, the $\partial u / \partial z$ variable can be eliminated.

In experimental studies, we have used the double angle method for $B_{1}$ mapping. In order to reduce the acquisition times, other $B_{1}$ mapping techniques such as actual flip angle imaging (AFI) [53], Multiple TR $\mathrm{B}_{1} / \mathrm{T}_{1}$ mapping (MTM) [52], and Bloch-Siegert shift based $B_{1}$ mapping [54] can be used. For example, Voigt et al. have used MTM and have achieved a total acquisition time of $16 \mathrm{~min}$ with eight averages in in vivo experiments [29]. Bulumulla et al. have used Bloch-Siegert shift based $B_{1}$ mapping [60]. We know from Sacolick et al., who have developed the Bloch-Siegert shift based $B_{1}$ mapping technique, that a single $B_{1}$ mapping can be completed in $18 \mathrm{~s}$ [54]. For the measurement of the phase of $B_{1}$ distributions we have used a SE sequence. However, Stehning et al. have used SSFP for phase measurement and this sequence takes less than a minute even for multi-slice and with multiple averaging [61]. In conclusion, significant reduction in total acquisition for complex $B_{1}$ map is possible and is essential for in vivo applications.

\section{CONCLUSION}

This study substantially improves the MREPT technique by proposing a novel method, cr-MREPT, which is capable of reconstructing conductivity and dielectric permittivity not only in regions where they vary slowly but also where they have high gradients. It has been shown that the proposed method is applicable in a standard MRI system using a quadrature birdcage coil. The method performs well against noise, against the transceive phase approximation and for complex electrical property distributions such as in the head. Further studies are needed to investigate the suitability of the cr-MREPT to real-life applications specifically in relation to padding structures and multi-coil configurations.

\section{REFERENCES}

[1] C. Gabriel, S. Gabriel, and E. Courhout, "The dielectric properties of biological tissues: I. Literature survey," Phys. Med. Biol., vol. 41, pp. 2231-2249, Nov. 1996.

[2] E. C. Fear, X. Li, S. C. Hagness, and M. A. Stuchly, "Confocal microwave imaging for the breast cancer detection: Localization of tumors in three dimensions," IEEE Trans. Biomed. Eng., vol. 49, no. 8, pp. 812-822, Aug. 2002.

[3] W. T. Joines, Y. Zhang, C. Li, and R. L. Jirtle, "The measured electrical properties of normal and malignant human tissues from 50 to 900 MHz," Med. Phys., vol. 21, pp. 547-550, Apr. 1994.

[4] A. J. Surowiec, S. S. Stuchly, J. B. Barr, and A. Swarup, "Dielectric properties of breast carcinoma and the surrounding tissues," IEEE Trans. Biomed. Eng., vol. 35, no. 4, pp. 257-263, Apr. 1988. 
[5] S. Gabriel, R. W. Lau, and C. Gabriel, "The dielectric properties of biological tissues: II. Measurements in the frequency range $10 \mathrm{~Hz}$ to 20 GHz," Phys. Med. Biol., vol. 41, pp. 2251-2269, Nov. 1996.

[6] L. T. Muftuler, M. Hamamura, O. Birgul, and O. Nalcioglu, "Resolution and contrast in magnetic resonance electrical impedance tomography (MREIT) and its application to cancer imaging," Technol. Cancer Res. Treat., vol. 3, pp. 599-609, Dec. 2004.

[7] V. Cherepenin, A. Karpov, A. Korjenevsky, V. Kornienko, A. Mazaletskaya, D. Mazourov, and D. Meister, "A 3-D electrical impedance tomography (EIT) system for breast cancer detection," Physiol. Meas., vol. 22, pp. 9-18, Feb. 2001.

[8] A. Romsauerova, M. Ewan, L. Horesh, T. I. Oh, E. J. Woo, and D. S. Holder, "A comparison of Multi-Frequency EIT systems intended for acute stroke imaging," in World Congress Med. Phys. Biomed. Eng., 2007.

[9] D. C. Barber and B. H. Brown, "Applied potential tomography," $J$. Phys. E: Sci. Instrum., vol. 17, no. 9, pp. 723-733, 1984.

[10] L. F. Fuks, M. Cheney, D. Isaacson, D. G. Gisser, and J. C. Newell, "Detection and imaging of electric conductivity and permittivity at low frequency," IEEE Trans. Biomed. Eng., vol. 38, no. 11, pp. 1106-1110, Nov. 1991.

[11] P. M. Edic, G. J. Saulnier, J. C. Newell, and D. Isaacson, "A real-time electrical impedance tomograph," IEEE Trans. Biomed. Eng., vol. 42, no. 9, pp. 849-859, Sep. 1995.

[12] N. G. Gençer, Y. Z. Ider, and S. J. Williamson, "Electrical impedance tomography: Induced-current imaging achieved with a multiple coil system," IEEE Trans. Biomed. Eng., vol. 43, no. 2, pp. 139-149, Feb. 1996.

[13] H. Griffiths, "Magnetic induction tomography," Meas. Sci. Technol., vol. 12, pp. 1126-1131, Aug. 2001.

[14] Y. Z. Ider and S. Onart, "Algebraic reconstruction for 3-D magnetic resonance-electrical impedance tomography (MREIT) using one component of magnetic flux density," Physiol. Meas., vol. 25, no. 1, pp. 281-294, Feb. 2004.

[15] O. Birgul and Y. Z. Ider, "Use of the magnetic field generated by the internal distribution of injected currents for electrical impedance tomography," presented at the 9th Int. Conf. Bio-Impedance, Heidelberg, Germany, 1995.

[16] Y. Z. Ider and O. Birgul, "Use of the magnetic field generated by the internal distribution of injected currents for electrical impedance tomography (MREIT)," Elektrik Turk. J. Electr. Comput. Sci., vol. 6, pp. $215-225,1998$.

[17] J. K. Seo, O. Kwon, and E. J. Woo, "Magnetic resonance electrical impedance tomography (MREIT); Conductivity and current density imaging," in J. Phys. Conf. Ser., 2005, vol. 12, pp. 140-155.

[18] E. J. Woo and J. K. Seo, "Magnetic resonance electrical impedance tomography (MREIT) for high-resolution conductivity imaging," Physiol. Meas., vol. 29, no. 10, pp. R1-R26, 2008.

[19] J. K. Seo, J. R. Yoon, E. J. Woo, and O. Kwon, "Reconstruction of conductivity and current density images using only one component of magnetic field measurements," IEEE Trans. Med. Imag., vol. 97, pp. 1121-1124, 2003.

[20] H. S. Nam, C. Park, and O. I. Kwon, "Non-iterative conductivity reconstruction algorithm using projected current density in MREIT," Phys. Med. Biol., vol. 53, pp. 6947-6961, 2008.

[21] G. C. Scott, M. L. G. Joy, R. L. Armstrong, and R. M. Henkelman, "Measurement of nonuniform current density by magnetic resonance," IEEE Trans. Med. Imag., vol. 10, no. 3, pp. 362-374, Sep. 1991.

[22] L. T. Muftuler and Y. Z. Ider, "Measurement of AC magnetic field distribution using magnetic resonance imaging," IEEE Trans. Med. Imag., vol. 16, no. 5, pp. 617-622, Oct. 1997.

[23] U. Mikac, F. Demsar, K. Beravs, and I. Sersa, "Magnetic resonance imaging of alternating currents," Magn. Reson. Med., vol. 19, pp. 845-856, 2001.

[24] E. M. Haacke, L. S. Petropoulos, E. W. Nilges, and D. H. Wu, "Extraction of conductivity and permittivity using magnetic resonance imaging," Phys. Med. Biol., vol. 38, no. 6, pp. 471-477, 1991.

[25] H. Wen, "Noninvasive quantitative mapping of conductivity and dielectric distributions using RF wave propagation effects in high-field MRI," in Proc. SPIE, 2003, vol. 471-477, p. 5030.

[26] U. Katscher, T. Voigt, C. Findeklee, P. Vernickel, K. Nehrke, and O. Dossel, "Determination of electrical conductivity and local SAR via B1 mapping," IEEE Trans. Med. Imag., vol. 28, no. 9, pp. 1365-1374, Sep. 2009.

[27] X. Zhang, S. Zhu, and B. He, "Imaging electric properties of biological tissues by RF field mapping in MRI," IEEE Trans. Med. Imag., vol. 29, no. 2, pp. 474-481, Feb. 2010.
[28] A. L. van Lier, D. O. Brunner, K. P. Pruessmann, D. W. Klomp, P. R. Luijten, J. J. Lagendijk, and C. A. van den Berg, "B1+Phase mapping at $7 \mathrm{~T}$ and its application for in vivo electrical conductivity mapping," Magn. Reson. Med., pp. 1-10, Mar. 2011.

[29] T. Voigt, U. Katscher, and O. Doessel, "Quantitative conductivity and permittivity imaging of the human brain using electric properties tomography," Magn. Reson. Med., vol. 66, no. 2, pp. 456-466, 2011.

[30] X. Zhang, P.-F. V. de Moortel, S. Schmitter, and B. He, "Complex B1 mapping and electrical properties imaging of the human brain using a 16-channel transceiver coil at 7 T," Magn. Reson. Med., vol. 69, no. 5, pp. 1285-1296, 2012.

[31] U. Katscher, C. Findeklee, and T. Voigt, "B1-based specific energy absorption rate determination for nonquadrature radiofrequency excitation," Magn. Reson. Med., vol. 68, no. 6, pp. 1911-1918, 2012.

[32] U. Katscher, T. Dorniok, C. Findeklee, P. Vernickel, and K. Nehrke, "In vivo determination of electric conductivity and permittivity using a standard MR system," presented at the IFMBE, Berlin, Germany, 2007.

[33] A. I. Nachman, D. Wang, W. Ma, and M. L. G. Joy, “A local formula for inhomogeneous complex conductivity as a function of the RF magnetic field," presented at the 15th Annu. Meet. ISMRM, Berlin, Germany, 2007.

[34] D. K. Sodickson, L. Alon, C. M. Deniz, R. Brown, B. Zhang, G. C. Wiggins, G. Y. Cho, N. B. Eliezer, D. S. Novikov, R. Lattanzi, Q. Duan, L. A. Sodickson, and Y. Zhu, "Local maxwell tomography using transmit-receive coil arrays for contact-free mapping of tissue electrical properties and determination of absolute RF phase," presented at the 20th Annu. Meet. ISMRM, Melbourne, Australia, 2012.

[35] D. K. Sodickson, L. Alon, C. M. Deniz, N. B. Eliezer, M. Cloos, L. A. Sodickson, M. C. Collins, G. C. Wiggins, and D. S. Novikov, "Generalized local maxwell tomography for mapping of electrical property gradients and tensors," presented at the 21th Annu. Meet. ISMRM, Salt Lake City, UT, 2013.

[36] S. Akoka, F. Franconi, F. Seguin, and A. le Pape, "Radiofrequency map of an NMR coil by imaging," Magn. Reson. Imag., vol. 11, pp. $437-441,1993$.

[37] J. K. Seo, M.-O. Kim, J. Lee, N. Choi, E. J. Woo, H. J. Kim, O. I. Kwon, and D.-H. Kim, "Error analysis of nonconstant admittivity for MR-based electric property imaging," IEEE Trans. Med. Imag., vol. 31, no. 2, pp. 430-437, Feb. 2012.

[38] D. I. Hoult, "The principle of reciprocity in signal strength calculations-A mathematical guide," Concepts Magn. Reson., vol. 12, pp. $173-187,2000$.

[39] F. A. Fernandez and L. Kulas, "A simple finite difference approach using unstructured meshes from FEM mesh generators," in Proc. IEEE 15th Int. Conf. Microw. Radar Wireless Commun., May 2004, vol. 2, pp. $585-588$.

[40] A. Savitzky and M. J. E. Golay, "Smoothing and differentiation of data by simplified least squares procedures," Analyt. Chem., vol. 36, no. 8, pp. $1627-1639,1964$

[41] N. Gurler and Y. Z. Ider, "FEM based design and simulation tool for MRI birdcage coils including eigenfrequency analysis," presented at the COMSOL, Milan, Italy, 2012.

[42] N. Gurler, F. S. Hafalir, O. F. Oran, and Y. Z. Ider, "A New accurate FEM based optimization method for birdcage coil design at high field strength," presented at the 21th Annu. Meet. ISMRM, Salt Lake City, UT, 2013.

[43] G. Scott, M. Joy, R. Armstrong, and R. Henkelman, "Sensitivity of magnetic-resonance current-density imaging," J. Magn. Reson., vol. 97, no. 2, pp. 235-254, Apr. 1992.

[44] H. Gudbjartsson and S. Patz, "The rician distribution of noisy MRI data," Magn. Reson. Med., vol. 34, no. 6, pp. 910-914, 1995.

[45] D. Tschumperle and R. Deriche, "Diffusion PDEs on vector-valued images," IEEE Signal Process. Mag., vol. 19, no. 5, pp. 16-25, Sep. 2002.

[46] A. Stogryn, "Equations for calculating the dielectric constant of saline water," IEEE Trans. Microw. Theory Tech., vol. 19, no. 8, pp. 733-736, Aug. 1971.

[47] E. Iwase, "Electrical conductivities of salt solutions containing agar," Bull. Chem. Soc. Jpn, vol. 2, no. 3, pp. 61-65, 1927.

[48] K. Iizuka, "An agar-agar chamber for the study of electromagnetic waves in an inhomogeneous medium," IEEE Trans. Antennas Propag., vol. 19 , no. 3, pp. 365-377, May 1971.

[49] R. Cooper, "The electrical properties of salt-water solutions over the frequency range 1-4000 Mc/s," J. Inst. Electr. Eng.-Part III: Radio Commun. Eng., vol. 93, no. 22, pp. 69-75, 1946. 
[50] M. J. Hamamura, L. T. Muftuler, O. Bilgul, and O. Nalcioglu, "Measurement of ion diffusion using magnetic resonance electrical impedance tomography," Phys. Med. Biol., vol. 51, no. 11, pp. 2753-2762, 2006.

[51] R. Stollberger and P. Wach, "Imaging of the active B1 field in vivo," Magn. Reson. Med., vol. 35, pp. 246-251, Feb. 1996.

[52] T. Voigt, K. Nehrke, O. Doessel, and U. Katscher, "T1 corrected B1 mapping using multi-TR gradient echo sequences," Magn. Reson. Med., vol. 64, pp. 725-733, Feb. 2010.

[53] V. L. Yarnykh, "Actual flip-angle imaging in the pulsed steady state: A method for rapid three-dimensional mapping of the transmitted radiofrequency field," Magn. Reson. Med., vol. 57, pp. 192-200, Jan. 2007.

[54] L. I. Sacolick, F. Wiesinger, I. Hancu, and M. W. Vogel, "B1 mapping by Bloch-Siegert shift," Magn. Reson. Med., vol. 63, no. 5, pp. 1315-1322, May 2010.

[55] W. Luo, M. T. Lanagan, C. T. Sica, Y. Ryu, S. Oh, M. Ketterman, Q. X. Yang, and C. M. Collins, "Permittivity and performance of dielectric pads with sintered ceramic beads in MRI: Early experiments and simulations at 3 T," Magn. Reson. Med., vol. 70, no. 1, pp. 269-275, 2013.
[56] M. Sreenivas, M. Lowry, P. Gibbs, M. Pickles, and L. Turnbull, "A simple solution for reducing artefacts due to conductive and dielectric effects in clinical magnetic resonance imaging at 3 T," Eur. J. Radiol., vol. 62, pp. 143-146, 2007.

[57] Y. Takayama, H. Nonaka, T. Obata, and H. Ikehira, "Reduction of a high-field dielectric artifact with homemade gel," Magn. Reson. Med. Sci., vol. 7, no. 1, pp. 37-41, 2008.

[58] O. F. Oran and Y. Z. Ider, "Magnetic resonance electrical impedance tomography (MREIT) based on the solution of the convection equation using FEM with stabilization," Phys. Med. Biol., vol. 57, pp. 5113-5140, 2012.

[59] F. S. Hafalir, O. F. Oran, N. Gurler, and Y. Z. Ider, "Magnetic resonance electrical properties tomography (MREPT) based on the solution of the convection-reaction equation," presented at the 21th Annu. Meet. ISMRM, Salt Lake City, UT, 2013.

[60] S. B. Bulumulla, T. B. Yeo, and Y. Zhu, "Direct calculation of tissue electrical parameters from B1 maps," presented at the 17th Annu. Meet. ISMRM, Honolulu, HI, 2009.

[61] C. Stehning, T. R. Voigt, and U. Katscher, "Real-time conductivity mapping using balanced SSFP and phase-based reconstruction," presented at the 19th Annu. Meet. ISMRM, Montreal, QC, Canada, 2011. 